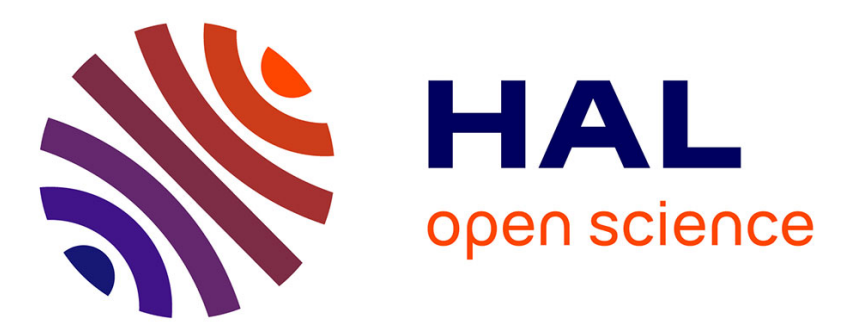

\title{
A feedback control perspective on biological control of dengue vectors by Wolbachia infection \\ Pierre-Alexandre Bliman
}

\section{To cite this version:}

Pierre-Alexandre Bliman. A feedback control perspective on biological control of dengue vectors by Wolbachia infection. European Journal of Control, 2020, 10.1016/j.ejcon.2020.09.006 . hal-03080967

\section{HAL Id: hal-03080967 https://hal.inria.fr/hal-03080967}

Submitted on 18 Dec 2020

HAL is a multi-disciplinary open access archive for the deposit and dissemination of scientific research documents, whether they are published or not. The documents may come from teaching and research institutions in France or abroad, or from public or private research centers.
L'archive ouverte pluridisciplinaire HAL, est destinée au dépôt et à la diffusion de documents scientifiques de niveau recherche, publiés ou non, émanant des établissements d'enseignement et de recherche français ou étrangers, des laboratoires publics ou privés. 


\title{
A Feedback Control Perspective on Biological Control of Dengue Vectors by Wolbachia Infection
}

This paper is dedicated to my mentor Michel Sorine, engineer and free spirit, untiring researcher and teacher, on the occasion of his 70th birthday

Pierre-Alexandre Bliman

Inria, Sorbonne Université, Université Paris-Diderot SPC, CNRS, Laboratoire

Jacques-Louis Lions, équipe MAMBA, F-75005 Paris, France; e-mail:

pierre-alexandre.bliman@inria.fr

\begin{abstract}
Controlling diseases such as dengue fever, chikungunya and zika fever by introduction of the intracellular parasitic bacterium Wolbachia in mosquito populations which are their vectors, is presently quite a promising tool to reduce their spread. While description of the conditions of such experiments has received ample attention from biologists, entomologists and applied mathematicians, the issue of effective scheduling of the releases remains an interesting problem. Having in mind the important uncertainties present in the dynamics of the two populations in interaction, we attempt here to identify general ideas for building feedback-based release strategies, enforceable to a variety of models and situations. These principles are exemplified by several feedback control laws whose stabilizing properties are demonstrated, illustrated numerically and compared, when applied to a model retrieved from [P.-A. Bliman et al., Ensuring successful introduction of Wolbachia in natural populations of Aedes aegypti by means of feedback control. J. of Math. Bio. 76(5):1269-1300, 2018]. The contribution is believed to be also of potential interest to tackle other important issues related to the biological control of vectors and pests. A crucial use of the theory of monotone dynamical systems is made in the derivations.
\end{abstract}

Keywords: Wolbachia, Global stabilization, Monotone dynamical systems, Bistable systems, Biological control 


\section{Introduction}

Already a major issue in southern countries since decades, the control of vector-borne diseases transmitted by mosquitoes recently became an important concern for northern countries too, due to the geographical dissemination of the vectors favored by climate change, urbanization and increasing international travel. When no vaccine or curative treatment exists, traditional control measures focus on reducing the vector population in order to reduce the pathogen transmission. Mechanical control methods by preventive actions and removal of breeding sites are invaluable, but costly and difficult to implement efficiently. Chemical control by insecticides has been quite popular, but on top of its negative impacts on humans and animals, it experiences decreasing efficiency due to resistance generation. Recently, various biological control methods have been proposed and tested as alternative or complementary strategies, typically by the release of transgenic or sterile mosquitoes [1, 2]. A new promising strategy based on the use of the bacterium Wolbachia, is being developed to control the spread of dengue fever and other diseases transmitted by species of the genus Aedes (chikungunya, zika fever, yellow fever). It is grounded in the fact that Wolbachia severely reduces the insect vectorial ability, indirectly by reducing lifespan and fertility, and directly by reducing the virus ability to proliferate within the organism [3, 4, 5, 6].

The dynamics resulting from the introduction of Wolbachia-infected mosquitoes in wild populations has been abundantly considered, even in the absence of spatial aspects; see [7, 8, 9, 10, 11, 12, 13, 14, 15, 16, 17], and [18, 19, 20] for the effects induced on a dengue epidemics. Field sampling methods allow to evaluate population density [21, 22, and such operations are commonly performed during experimental campaigns. Also, the presence of the bacterium in the captured sample may be investigated by polymerase chain reaction (PCR) method [5]. Theoretically, this opens up the possibility to assess the released quantities on the basis of the observed population, and to benefit from the multiple advantages of closed-loop methods. Most papers analyze only the effect of a unique, initial, release. By contrast, [11] considers continuous releases and [12] impulsive releases, both with constant release amplitude, while [16] provides a single linear control-law and [14, 15] optimal control. However these stabilization results are highly dependent upon the setting, and their generalization is in no way straightforward. Our aim here is to identify simple feedback control principles, based on the understanding of 
the biological phenomena involved, and to test them on the model introduced in [16]. For this purpose we propose, analyze and simulate several control laws achieving complete infestation, through the release of infected adults or infected larvae. Biological control is the use of living organisms or their products to control vector and pest insects [23, 24, 25, 26]. Apart from the specific issue considered here, we believe that the feedback-control principles displayed in this work may be of broader interest for application of Control theory in this domain of growing importance.

The content of the paper is as follows. The characteristics of the compartmental model developed in [16] are recalled in Section 2, together with some analysis results. Three independent control principles are exposed in Section 3. materialized in several feedback control laws provided in Theorems 5, 6, and 7, and illustrated by numerical simulations. In order to avoid large peak values of the control, we then study the issue of saturated control laws in Section 4 (Theorem 9) and provide comparison with the previous numerical results. The control laws in Theorems 5,6 and 7 are constrained by inequalities: they achieve the desired objective provided that their value is taken at least equal to certain function of the current state. In order to incorporate situations where the latter is not fully measured, we propose in Section 5 a class of interval observers [27, 28, 29] for the studied model, allowing for the use of the previous results for the design of output feedback control laws. Concluding remarks are given in Section 6. For better readability, the demonstrations are gathered in Appendix A.

It is worth pointing out that most of the arguments in this paper are based on Monotone systems theory [30, 31]. A preliminary version of this paper has been presented to European Control Conference, Napoli (Italy), June 25-28, 2019 [32].

Notations and abbreviations. The following notations are used for the positive, resp. negative, parts of a real number $z:|z|_{+}:=\max \{z, 0\},|z|_{-}:=$ $-\min \{z, 0\}$. Both numbers are nonnegative, and $z=|z|_{+}-|z|_{-}$for any $z \in \mathbb{R}$. The symbol $\mathbb{R}_{+}$, resp. $\mathbb{R}_{-}$, denotes the set of the nonnegative, resp. nonpositive, real numbers.

The usual order relation $\geqslant$ in $\mathbb{R}$ is employed, and the same notation is extended to vectors and matrices by the product order: $\left(x_{1}, x_{2}\right) \geqslant 0$ iff $x_{1} \geqslant 0$ and $x_{2} \geqslant 0$. (Notice that a different order relation, denoted $\geqslant_{C}$, is defined and used latter in the text: the two relations should not be confused.) It is similarly extended to locally integrable functions taking on values in one of 
these ordered spaces, with the evident meaning. In all cases, the symbol $>$ is used as usual to mean 'at most equal to, but not equal'. As an example, for locally integrable functions $f, g$ defined on a common domain, ' $f>g$ ' is equivalent to: ' $f \geqslant g$ and $f>g$ on a nonzero-measured set'.

Whenever the sign \pm is used, it means that the relevant formula is valid for + and for - . Usual matrix notations are employed: $I_{n}, 0_{n}$ denote respectively the identity and null square matrices of order $n, n \in \mathbb{N},{ }^{\top}$ denotes transposition. Last, we use in the text few standard abbreviations: GAS (Globally Asymptotically Stable), LAS (Locally Asymptotically Stable) and SOP (Strongly-Order Preserving).

\section{Modeling}

\subsection{Model equations, well-posedness and invariant set}

The compartmental model in [16] focuses on the main effects pertinent for control purposes. It contains two life phases: a preliminary phase which gathers the early, aquatic, stages (egg, larva and pupa), subject to competition; and an adult, aerial, mature phase. The corresponding state variables are respectively denoted $L$ ('larvae') and $A$ ('adults'), and the uninfected and Wolbachia-infected populations are distinguished by indices $U$ and $W$. Assuming in first approximation that the sex ratio is stationary and the mortality is sex-independent, these variables represent indifferently the quantities of males or females, up to constant ratio. After normalization! the 4-dimensional population model used in the present paper is as follows [16]:

$$
\begin{gathered}
\dot{L}_{U}=\gamma_{U} \mathcal{R}_{U} \frac{A_{U}}{A_{U}+A_{W}} A_{U}-\left(1+L_{U}+L_{W}\right) L_{U} \\
\dot{A}_{U}=L_{U}-\gamma_{U} A_{U} \\
\dot{L}_{W}=\gamma_{W} \mathcal{R}_{W} A_{W}-\left(1+L_{U}+L_{W}\right) L_{W}+u_{L}(t) \\
\dot{A}_{W}=L_{W}-\gamma_{W} A_{W}+u_{A}(t)
\end{gathered}
$$

The function $\mathbb{R}_{+} \times \mathbb{R}_{+} \rightarrow \mathbb{R}_{+},\left(A_{U}, A_{W}\right) \mapsto \frac{A_{U}}{A_{U}+A_{W}} A_{U}$ that appears in (1a) is not defined for $\left(A_{U}, A_{W}\right)=(0,0)$. However taking the value 0 at this

\footnotetext{
${ }^{1}$ The state and time variables are dimensionless in the new setting. The latter is chosen such that the basic larval mortality rate is equal to one (normalised) individual per normalised time unit. See [16] for details.
} 
point yields a continuous and locally Lipschitz extended function. The term $u_{L}$ (resp. $u_{A}$ ) represents rate of release of the infected larvae (resp. adults) per time unit to install Wolbachia infection in the population. By construction both signals have nonnegative values. Using $x:=\left(L_{U}, A_{U}, L_{W}, A_{W}\right), u:=$ $\left(u_{L}, u_{A}\right)$ as state and input variables, the previous controlled system writes compactly:

$$
\dot{x}=f(x)+B u, \quad B:=\left(\begin{array}{cccc}
0 & 0 & 1 & 0 \\
0 & 0 & 0 & 1
\end{array}\right)^{\top},
$$

where the function $f$ is easily expressed from (1).

We call admissible inputs any $u(t)$ locally integrable with respect to time and taking on almost everywhere nonnegative values. Well-posedness of the Cauchy problem for system (1) with any admissible input does not present specific difficulty, and the corresponding solution has nonnegative components [16, Theorem 1].

\subsection{Phenomenological hypotheses on the infection dynamics}

To recap, the main phenomenological assumptions contained in the previous infection model are the following.

1. The Wolbachia infection in Aedes mosquito leads to lifespan shortening through adult mortality increase [3, 33] and fecundity rate reduction [34. This is accounted for by the following inequalities between normalized constants:

$$
0<\gamma_{U}<\gamma_{W}, \quad \mathcal{R}_{U}>\mathcal{R}_{W}>1 .
$$

The constant $\gamma_{\eta}, \eta=U, W$, is linked to the adult mortality, while $\mathcal{R}_{\eta}$ is the basic offspring number of each isolated population. Both isolated populations are sustainable, with a greater basic offspring number for the uninfected population, in accordance with the fact that Wolbachia globally reduces the fitness of the infected mosquitoes. On the other hand, the difference of larval development time and mortality between the two populations is regarded as limited and is not modeled here.

2. The density-dependent mortality is a major component of larval dynamics [35, 36], rendered here by quadratic competition terms in the immature phase dynamics, identical for each population. Accounting for restricted food and space in the breeding sites, it acts evenly on the immature infected and uninfected populations, with a rate proportional to its global size $L_{U}+L_{W}$. 
3. The Wolbachia transmission occurs only vertically: from mother to offspring. It is accompanied by a phenomenon called cytoplasmic incompatibility, which provides a reproduction advantage to infected females against uninfected ones and facilitates the spread of the bacterium. Cytoplasmic incompatibility is the fact that an uninfected female only produces offspring when mating with an uninfected male [37, 3, 33, 38]. The probability of a male being uninfected is $\frac{A_{U}}{A_{U}+A_{W}}$, and the nonlinear birth term $\frac{A_{U}}{A_{U}+A_{W}} A_{U}$ in (1a) accounts for such encounters. On the contrary when an infected female mates, the outcome is infected independently of whether the male is infected or not. By contrast with (1a), the birth term for the infecteds in (1c) is simply proportional to the corresponding population $A_{W}$.

Throughout the remainder of this paper, we assume that assumption (3) holds.

\subsection{Analysis of the uncontrolled model}

Before recalling the key results on the uncontrolled model (1) with $u_{L}=$ $u_{A} \equiv 0$, we state an elementary result that describes the behaviour of each population when isolated.

Theorem 1 (A one-population auxiliary system). For any given $\gamma, \mathcal{R}>0$, the system

$$
\dot{L}=\gamma \mathcal{R} A-(1+L) L, \quad \dot{A}=L-\gamma A
$$

admits a unique nonnegative solution for any nonnegative initial condition, and this solution is uniformly ultimately bounded. Moreover, (4) fulfils the following properties.

- If $\mathcal{R} \leqslant 1$, then (4) admits only the extinction equilibrium $x_{0}^{*}:=(0,0)$, and the latter is GAS.

- If $\mathcal{R}>1$, then (4) also admits the positive equilibrium $x^{*}=\left(L^{*}, A^{*}\right)$ defined as

$$
L^{*}:=\mathcal{R}-1, \quad A^{*}:=\frac{L^{*}}{\gamma}
$$

which is LAS and attracts every nonzero trajectory. 
Theorem 1 enlightens the critical importance of $\mathcal{R}$ in the viability of the population. Its proof is elementary, and provided for sake of completeness in Appendix A.1, as an introduction to the forthcoming monotonicity arguments.

Before examining the situation of the complete uncontrolled system (1), we introduce an instrumental ordering of the state space $\mathbb{R}_{+}^{4}$, called competition partial order after [39].

Definition 1 (Competition partial order relation). Let $\geqslant_{C}$ be the ordering induced on $\mathbb{R}^{4}$ by the orthant $\mathbb{R}_{-} \times \mathbb{R}_{-} \times \mathbb{R}_{+} \times \mathbb{R}_{+}$, that is

$$
x \geqslant_{C} x^{\prime} \Leftrightarrow x_{i} \leqslant x_{i}^{\prime}, i=1,2 \text { and } x_{i} \geqslant x_{i}^{\prime}, i=3,4 .
$$

Similarly, one defines the reverse order relation $\leqslant_{C}$ :

$$
x \leqslant_{C} x^{\prime} \Leftrightarrow x^{\prime} \geqslant_{C} x \Leftrightarrow x_{i}^{\prime} \leqslant x_{i}, i=1,2 \text { and } x_{i}^{\prime} \geqslant x_{i}, i=3,4 .
$$

The order intervals associated to this ordering are then defined as in [31]: for any $x_{*}, x^{*} \in \mathbb{R}^{4}$,

$$
\llbracket x_{*} ; x^{*} \rrbracket:=\left\{x \in \mathbb{R}^{4}: x_{*} \leqslant_{C} x \leqslant_{C} x^{*}\right\} .
$$

In other words, $x \in \llbracket x_{*} ; x^{*} \rrbracket$ iff $x_{* i} \geqslant x_{i} \geqslant x_{i}^{*}$ for $i=1,2$ and $x_{* i} \leqslant x_{i} \leqslant x_{i}^{*}$ for $i=3,4$. One denotes $>_{C}$ the strict order relation associated to $\geqslant_{C}$. In other words, $x>_{C} x^{\prime}$ means: $x \geqslant_{C} x^{\prime}$, but $x \neq x^{\prime}$. Also, one defines $x \gg_{C} x^{\prime}$ as: $x-x^{\prime} \in$ int $\mathbb{R}_{-} \times \mathbb{R}_{-} \times \mathbb{R}_{+} \times \mathbb{R}_{+}$, that is $x_{i}<x_{i}^{\prime}, i=1,2$ and $x_{i}>x_{i}^{\prime}$, $i=3,4$.

Transposing Theorem 1 discloses immediately the existence, under hypothesis (3), of two equilibria, characterized by the state values

$$
x_{U}^{*}:=\left(L_{U}^{*}, A_{U}^{*}, 0,0\right) \quad \text { and } \quad x_{W}^{*}:=\left(0,0, L_{W}^{*}, A_{W}^{*}\right),
$$

where:

$$
L_{\eta}^{*}:=\mathcal{R}_{\eta}-1, \quad A_{\eta}^{*}:=\frac{L_{\eta}^{*}}{\gamma_{\eta}}, \quad \eta=U, W .
$$

They correspond respectively to disease-free and complete infestation situations. Being composed solely of uninfected or of infected mosquitoes, we call homogeneous these two equilibria. A complete picture is provided by the following result, which summarizes and slightly extends results from [16, Theorems 1 and 7]. It establishes that system (1) presents bistability: the purpose of the control considered later is typically to pass from the diseasefree situation to complete infestation. A rapid proof of Theorem 2 is given in Appendix A.2, see more details in [16]. 
Theorem 2 (Bistability of the uncontrolled system). System (1) with zero control $(u \equiv 0)$ admits, for any nonnegative initial condition, a unique nonnegative solution, and this solution is uniformly ultimately bounded. Moreover, it fulfils the following properties.

- The uncontrolled system (1) possesses four equilibrium points: two homogeneous equilibria, namely the Wolbachia free equilibrium $x_{U}^{*}$ and the complete Wolbachia infection equilibrium $x_{W}^{*}$; the extinction equilibrium $(0,0,0,0)$; and a positive coexistence equilibrium $x_{U W}^{*}$.

- The homogeneous equilibria are ordered according to $x_{U}^{*} \leqslant_{C} x_{W}^{*}$, and the two other ones pertain to the order interval $\llbracket x_{U}^{*} ; x_{W}^{*} \rrbracket$. The former ones are LAS, while the extinction equilibrium is repelling ${ }^{2}$ and the coexistence equilibrium is unstable.

- The union of the basins of attraction of the two LAS equilibria is open and dense in $\mathbb{R}_{+}^{4}$.

The monotonicity properties being central for all the subsequent analysis, we recall here some definitions, borrowed from [31].

Definition 2 (Monotone semiflows). Let $\Phi: \mathbb{R}_{+} \times X \rightarrow X$ denote a continuous semiflow in a metric space $X$ endowed with an order relation $\geqslant$ generated by a cone $X_{+} . \Phi$ is called monotone provided that

$$
\Phi_{t}(x) \geqslant \Phi_{t}(y) \quad \text { whenever } \quad x \geqslant y \text { and } t \geqslant 0
$$

$\Phi$ is called strongly order-preserving (SOP) if it is monotone and whenever $x>y$ there exist open neighbourhoods $U_{x}, U_{y}$ of $x$ and $y$ in $X$, and $t \geqslant 0$, such that

$$
\Phi_{t}\left(U_{x}\right) \geqslant \Phi_{t}\left(U_{y}\right)
$$

Last, $\Phi$ is called strongly monotone if ${ }^{3}$

$$
\Phi_{t}(x) \gg \Phi_{t}(y) \quad \text { whenever } \quad x>y \text { and } t>0 \text {. }
$$

\footnotetext{
${ }^{2}$ that is [39], there exists a pointed neighbourhood $U$ of this point such that: $\forall x \in$ $U, \exists t>0, \Phi_{t}(x) \notin U$, where $\Phi_{t}(x)$ designates the value at time $t$ of the solution leaving $x$ at time 0 .

${ }^{3}$ By definition, for any $x, y \in X, x \gg y$ if $x-y$ pertains to the interior int $X_{+}$.
} 
Notice that for a SOP semiflow $\Phi$, one has, using the notations of the definition, $\Phi_{t^{\prime}}\left(U_{x}\right) \geqslant \Phi_{t^{\prime}}\left(U_{y}\right)$ for any $t^{\prime} \geqslant t$.

The previous setting permits to state now a key property of system (1).

Theorem 3 (Strong order-preservation for the uncontrolled system (1)). System (1) with zero input $u$ is SOP in $\mathbb{R}_{+}^{4}$ with respect to the competition partial order (5).

This result is demonstrated in [16, Theorem 5]. We provide here a quick proof, for sake of completeness. The reader is referred to the original article for complete details.

Proof. The system is shown to be monotone with respect to the order (5) by checking the Kamke's conditions adapted to the order relation generated by an orthant (see e.g. [40, Corollary III.3]), namely $\varepsilon_{i} \varepsilon_{j} \frac{\partial f_{i}}{\partial x_{j}} \geqslant 0$, with here $\varepsilon_{i}=-1, i=1,2$ and $\varepsilon_{i}=1, i=3,4$. Moreover the Jacobian matrix is irreducible if $A_{U} \neq 0$ and $A_{W} \neq 0$. Using the fact that the 2-dimensional flow restricted to the subspace $\left\{x \in \mathbb{R}_{+}^{4}: L_{U}=A_{U}=0\right\}$ is strongly monotone (and similarly for the 2-dimensional flow restricted to $\left\{x \in \mathbb{R}_{+}^{4}\right.$ : $\left.\left.L_{W}=A_{W}=0\right\}\right)$, one concludes that the flow is SOP in $\mathbb{R}_{+}^{4}[16$.

\subsection{Forward-invariance of the controlled system}

As a result of the nonnegativeness of the input values, one has the following invariance result for equation (1) which will be useful in the sequel.

Theorem 4 (A positive invariant set). For any admissible input $u$, the set $\mathcal{S}:=\left\{x=\left(L_{U}, A_{U}, L_{W}, A_{W}\right) \in \mathbb{R}_{+}^{4}: L_{U} \leqslant L_{U}^{*}, A_{U} \leqslant A_{U}^{*}\right\}$ is positivelyinvariant.

Proof. The proof relies on the fact that, for any $x \in \mathcal{S}$, one has $(f(x))_{i} \leqslant 0$ whenever $i=1$ and $L_{U}=L_{U}^{*}$, or $i=2$ and $A_{U}=A_{U}^{*}$.

\section{Feedback control principles}

Our aim in this paper is to encounter state feedback laws guaranteeing the elimination of the uninfected population of mosquitoes and its replacement by a Wolbachia-infected population, through adequate releases of larvae or adults. This is seen in the sequel as a problem of global stabilization of the complete infection equilibrium $x_{W}^{*}$. Due to the uncertainty inherent to the models of population dynamics, it is quite valuable to have for this purpose 
feedback control strategies obeying general principles, "independent of the model'. The results given below all provide prescribed feedback value, with the property that any input signal taking on values larger or equal achieves stabilization - a feature believed to offer robustness of the proposed control laws with respect to uncertainty. In particular, these results may be easily adapted to be used with upper and lower estimates of the state variable, instead of the state variable itself. A class of interval observers that perform this task when only part of the state is available for measurement is presented in Section 5, allowing to design output feedback laws from the present work.

We first expose in Section 3.1 a method of control by release of larvae, then two related methods of control by release of adult mosquitoes in Sections 3.2 and 3.3 . These results are illustrated by numerical essays in Section 3.4 .

\subsection{Control by release of larvae through compensation}

We first examine control based on release of larvae. This method amounts to acting on the input term $u_{L}$ in $(1 \mathrm{c})$. In view of $(1 \mathrm{c})-(1 \mathrm{~d})$, the only hindrance to natural growth of the infected population lies on the competition term in the previous equation. Therefore,

If the rate of introduction of infected larvae $u_{L}$ in $(1 \mathrm{c})$ is always sufficient to compensate for the competition effect, then the infected population should evolve unconstrained and reach the complete infestation equilibrium, inducing collapse of the uninfected population through the cytoplasmic incompatibility and competitive pressure terms in (1a).

This is evidenced by the following result, whose proof is given in Appendix A.3.

Theorem 5 (Sufficient conditions for successful introduction via release of larvae). Assume that for some $T \geqslant 0$,

$$
u_{L}>L_{U} L_{W}, \quad u_{A} \geqslant 0 \quad \text { on }[T,+\infty)
$$

Then

$$
\lim _{t \rightarrow+\infty}\left(\begin{array}{l}
L_{U}(t) \\
A_{U}(t)
\end{array}\right)=\left(\begin{array}{l}
0 \\
0
\end{array}\right), \liminf _{t \rightarrow+\infty}\left(\begin{array}{c}
L_{W}(t) \\
A_{W}(t)
\end{array}\right) \geqslant\left(\begin{array}{c}
L_{W}^{*} \\
A_{W}^{*}
\end{array}\right)
$$

If moreover $u_{L}, u_{A}$ vanish when $t \rightarrow+\infty$, then (6) holds with an equality. 
Recall that the strict inequality in $(\mathbf{L})$ has to be interpreted according to the quote in the Notations paragraph (Section 1), as: $u_{L}(t) \geqslant L_{U}(t) L_{W}(t)$, with inequality on a nonzero-measured set. If e.g. $u_{L}(t)=L_{U}(t) L_{W}(t)$ for any large enough time $t$, then stabilization is realized with vanishing control input.

Whenever upper and lower state estimates of the solution of (1) are available (provided e.g. by an interval observer, as presented in Section 5 below), then the first part of $(\mathbf{L})$ is obviously fulfilled if

$$
u_{L}>L_{U}^{+} L_{W}^{+} \text {on }[T,+\infty),
$$

with $L_{\eta}^{+}(t) \geqslant L_{\eta}(t), \eta=U, W$, for any $t \geqslant T$.

Remark 1. Notice that (6) may be expressed equivalently as

$$
\liminf _{t \rightarrow+\infty} x(t) \geqslant_{C} x_{W}^{*}
$$

where $\lim _{\text {inf }}$ represents the limit inferior in the sense of the order relation $\geqslant_{C}$ defined in (5).

\subsection{Control by release of adults through fitness dominance}

We now consider release of adult mosquitoes. As a preliminary, notice that, if the inter-species competition induced by the presence of infected mosquitoes is sufficient to make unviable the uninfected population, then the latter disappears. However, adults do not interact directly, as testified by equations $1 \mathrm{~b}$ and $(1 \mathrm{~d})$; but they participate centrally to the natality. With this in mind,

If, due to releases of adults through the input $u_{A}$ in $(1 \mathrm{~d})$, the 'apparent' basic offspring number $\mathcal{R}_{U} \frac{A_{U}}{A_{U}+A_{W}}$ of the uninfected population is always kept smaller than 1 (or equivalently if $\frac{A_{W}}{A_{U}}$ is kept larger than $\left.\mathcal{R}_{U}-1\right)$, then the uninfected population should evolve sensibly as in equation (4) - however with a subcritical 'apparent' basic offspring number, therefore inducing collapse.

This suggests that targeting the ratio $\frac{A_{W}}{A_{U}}$ could be a general way to eliminate the uninfected population. In order to enforce that $\frac{A_{W}}{A_{U}} \geqslant k_{U}$ asymptotically for some $k_{U}>\mathcal{R}_{U}-1$, the idea in the following result is simply to impose, for some $k>0$, the 1st-order evolution

$$
\frac{d\left(A_{W}-k_{U} A_{U}\right)}{d t}+k\left(A_{W}-k_{U} A_{U}\right) \geqslant 0 .
$$


Theorem 6 (Sufficient conditions for successful introduction via release of adults -1 st method). Assume that for given constants $k>0, k_{U}>\mathcal{R}_{U}-1$ and for some $T \geqslant 0$,

$$
u_{L} \geqslant 0, u_{A}>w^{\top} x \text { on }[T,+\infty), w^{\top}:=\left(\begin{array}{llll}
k_{U} & k_{U}\left(k-\gamma_{U}\right) & -1 & \left(\gamma_{W}-k\right)
\end{array}\right)
$$

Then all conclusions of Theorem 5 hold.

A full proof of Theorem 6 is presented in Appendix A.4. Notice that, due to (3), the vector $w$ defined in the statement fulfils $w<_{C} 0$ whenever $k \geqslant \gamma_{W}$. An important feature is that in such case,

$$
\limsup _{t \rightarrow+\infty} w^{\top} x(t) \leqslant 0
$$

when (6) is fulfilled. Therefore, if $u_{A}(t)=\left|w^{\top} x(t)\right|_{+}$for large enough time, then the control input indeed vanishes asymptotically.

Just as (7) was sufficient to guarantee $(\mathbf{L})$, when upper and lower estimates $L_{\eta}^{-}(t) \leqslant L_{\eta}(t) \leqslant L_{\eta}^{+}(t), A_{\eta}^{-}(t) \leqslant A_{\eta}(t) \leqslant A_{\eta}^{+}(t), \eta=U, W$ are available for any $t \geqslant T$, one may as well replace the second part of $(A)$ by a (stronger) sufficient condition based on these estimates. When e.g. $k \geqslant \gamma_{W}$, this condition writes

$$
u_{A}>k_{U} L_{U}^{+}+k_{U}\left(k-\gamma_{U}\right) A_{U}^{+}-L_{W}^{-}-\left(k-\gamma_{W}\right) A_{W}^{-} .
$$

\subsection{Control by release of adults through fitness dominance}

The previous result was obtained by having the 'apparent' basic offspring number $\mathcal{R}_{U} \frac{A_{U}}{A_{U}+A_{W}}$ of the uninfected population smaller than 1 . We here refine this argument. Indeed,

If the 'apparent' basic offspring number $\mathcal{R}_{U} \frac{A_{U}}{A_{U}+A_{W}}$ is always smaller than the basic offspring number $\mathcal{R}_{W}$ of the infected population, then the former should grow more slowly than the latter one, and collapse if the total population remains bounded.

This is what is exploited in the following result, proved in Appendix A.5.

Theorem 7 (Sufficient conditions for successful introduction via release of adults - 2nd method). Assume that (A) holds for given constants $k>0$, $k_{U}>\frac{\mathcal{R}_{U}}{\mathcal{R}_{W}}-1$ (instead of $\mathcal{R}_{U}-1$ ) and for some $T \geqslant 0$. Then all conclusions of Theorem 5 hold. 
Due to the fact that $\mathcal{R}_{W}>1$, the hypothesis on $k_{U}$ in Theorem 7 is weaker than in Theorem 6, so Theorem 7 is stronger. The remarks made in the sequel of Theorem 6 are also valid for Theorem 7 .

Remark 2. One may notice the proximity in spirit between the guiding principles of Theorems 6 and 7, and the notion of variable structure system [42, 43]: the control seeks to create fast transient targeting the 'sliding region' $A_{W}-\left(\mathcal{R}_{U}-1\right) A_{U} \geqslant 0$ or $A_{W}-\left(\mathcal{R}_{U} / \mathcal{R}_{W}-1\right) A_{U} \geqslant 0$; and then a slower, ultimately uncontrolled, motion occurs in order to reach the desired equilibrium.

\subsection{Numerical simulations - I}

The following numerical values, extracted from [16], have been adopted: $\gamma_{U}=0.79365, \gamma_{W}=0.99207, \mathcal{R}_{U}=45, \mathcal{R}_{W}=34.2$, so that $L_{U}^{*}=44.0, A_{U}^{*}=$ 55.4, $L_{W}^{*}=33.2, A_{W}^{*}=33.5$. All essays have been produced with the help of the stiff option of the ode function of the free open-source software Scilab. We show here numerical simulations of the system (2) with the control laws designed in Theorems 5, 6 and 7. In order to concentrate on the principles of the feedback, only state feedback simulations have been performed, essays involving output feedback laws may be found in [32, 41]. The initial state is systematically taken at the disease-free equilibrium $\left(L_{U}^{*}, A_{U}^{*}, 0,0\right)$. All essays show, as expected, convergence of the controlled system towards the complete infestation equilibrium $\left(0,0, L_{W}^{*}, A_{W}^{*}\right)$.

\subsubsection{Releases of larvae (Theorem 5)}

The control $u:=\left(u_{L}, u_{A}\right)$ is defined here by taking equalities in $(\mathbf{L})$. In order to obtain the strict inequality in $(\mathbf{L})$, a small nonzero initialization of $L_{W}$ is imposed $\left(L_{W}(0)=10^{-4}\right)$, emulating an initial impulsive release. The evolution of the populations is shown in Figure 1, while in Figure 2 is shown the evolution of the input variable $u_{L}(t)$.

The total number of mosquitoes $\int_{0}^{+\infty} u_{L}(t) \cdot d t$ needed to reach complete infection is given in Table 1. Recall that this is a normalized number.

\begin{tabular}{|l|l|}
\hline Total number of released larvae & $2.17 \cdot 10^{3}$ \\
\hline
\end{tabular}

Table 1: Number of released larvae until complete infection for the non-saturated control (see Section 3.4.1) 

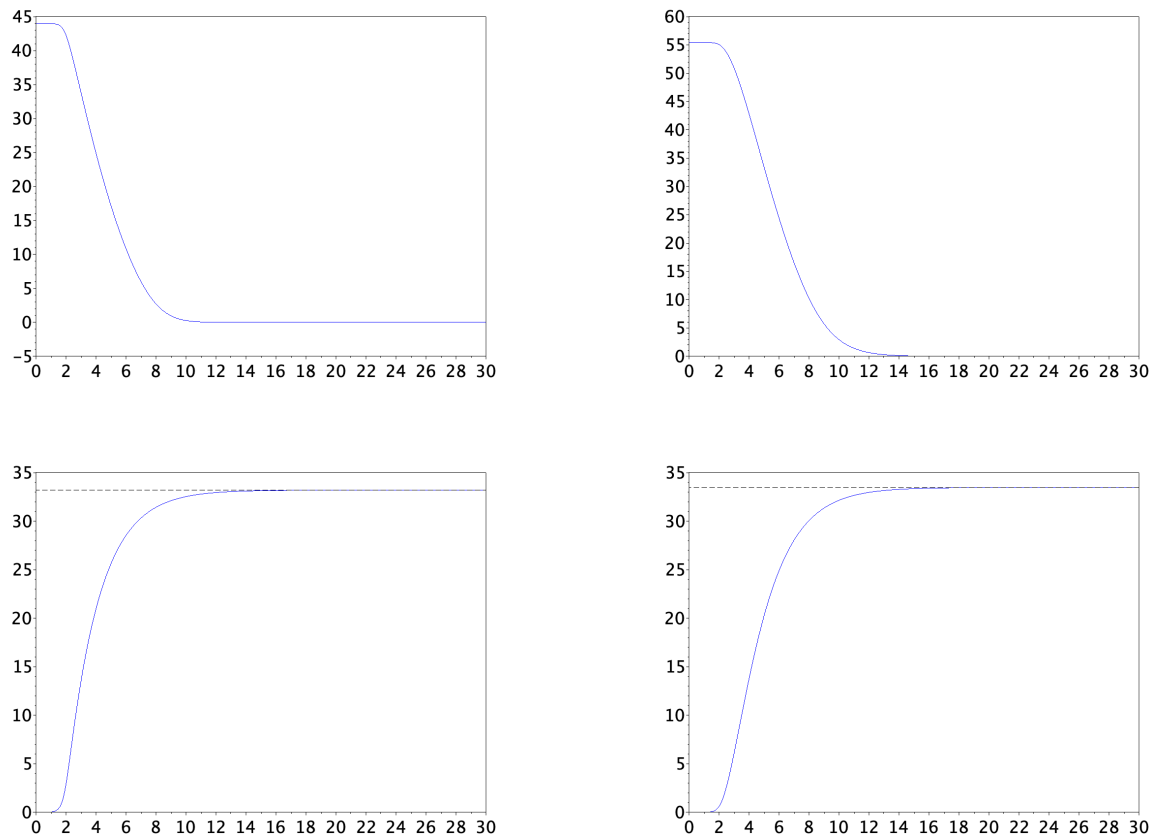

Figure 1: Release of larvae. Evolution of the uninfecteds (top) and Wolbachia-infecteds (bottom), as functions of time. The larvae appear on the left column, the adults on the right one. The horizontal dotted lines show the asymptotic values at the complete infection equilibrium.

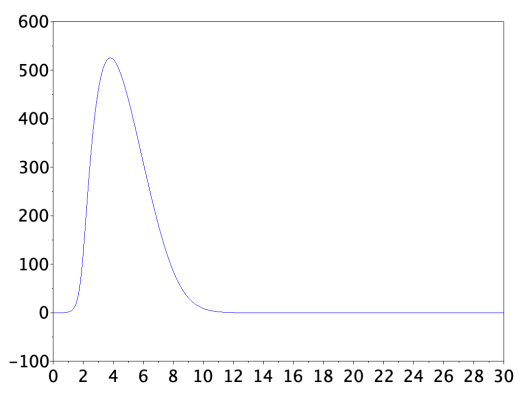

Figure 2: Release of larvae. Evolution of the control input $u_{L}$ as function of time. 


\subsubsection{Releases of adult mosquitos (Theorems 6 and 7)}

The control $u:=\left(u_{L}, u_{A}\right)$ is defined by taking equalities in the formulas in A, more precisely $u_{L}=0, u_{A}=\left|w^{\top} x\right|_{+}$. One chooses $k=\gamma_{W}$, thus letting $w^{\top}:=\left(\begin{array}{llll}k_{U} & k_{U}\left(\gamma_{W}-\gamma_{U}\right) & -1 & 0\end{array}\right)$. The evolution of the populations are shown in Figure 3 for the case $k_{U}=1.1\left(\mathcal{R}_{U}-1\right)$ (Theorem 6 ). One shows in Figure 4 the evolution of $A_{W}-k_{U} A_{U}$, and the value of the signal $w^{\top} x$, from which may be deduced the evolution of the input $u_{A}=\left|w^{\top} x\right|_{+}$. The instants where these two signals cancel are shown. As is evident, the input $u_{A}$ vanishes in finite time. Notice the rapid decrease of the uninfected population, and the large overshoots visible in the infected ones, before the settling of the asymptotic regime. The total number of adults released $\int_{0}^{+\infty} u_{A}(t) \cdot d t$ is given in Table 2.

The same information corresponding to the case $k_{U}=1.01\left(\mathcal{R}_{U} / \mathcal{R}_{W}-1\right)$ (Theorem 7) are provided in Figures 5 and 66. The establishment of the infection is sensibly slower than in the previous case. However, the input effort is much smaller, as shown by comparing the peak values (see Figures 4 and 6), as well as the total numbers of released adults in Table 2 .

\begin{tabular}{|l|l|}
\hline Total number of released adults, $k_{U}=1.01\left(\mathcal{R}_{U}-1\right)$ & $5.69 \cdot 10^{2}$ \\
\hline Total number of released adults, $k_{U}=1.01\left(\mathcal{R}_{U} / \mathcal{R}_{W}-1\right)$ & $1.98 \cdot 10$ \\
\hline
\end{tabular}

Table 2: Number of released adults until complete infection for the non-saturated control (see Section 3.4.2

\section{Saturated feedback control}

The previous results and simulations demonstrated the relevance of the proposed infection methods. With the purpose of reducing the maximal release rate, and possibly the total number of released mosquitoes, we explore now the possibility to reach full infection while imposing saturation to the previous control strategies. We first determine in Section 4.1 the least constant release values that ensure remotion of the uninfecteds. We then investigate in Section 4.2 the design of saturated feedback control, in an attempt to merge the results of Sections 3 and 4.1 . Corresponding numerical simulations are shown in Section 4.3 and compared with the simulations depicted in Section 3.4. 

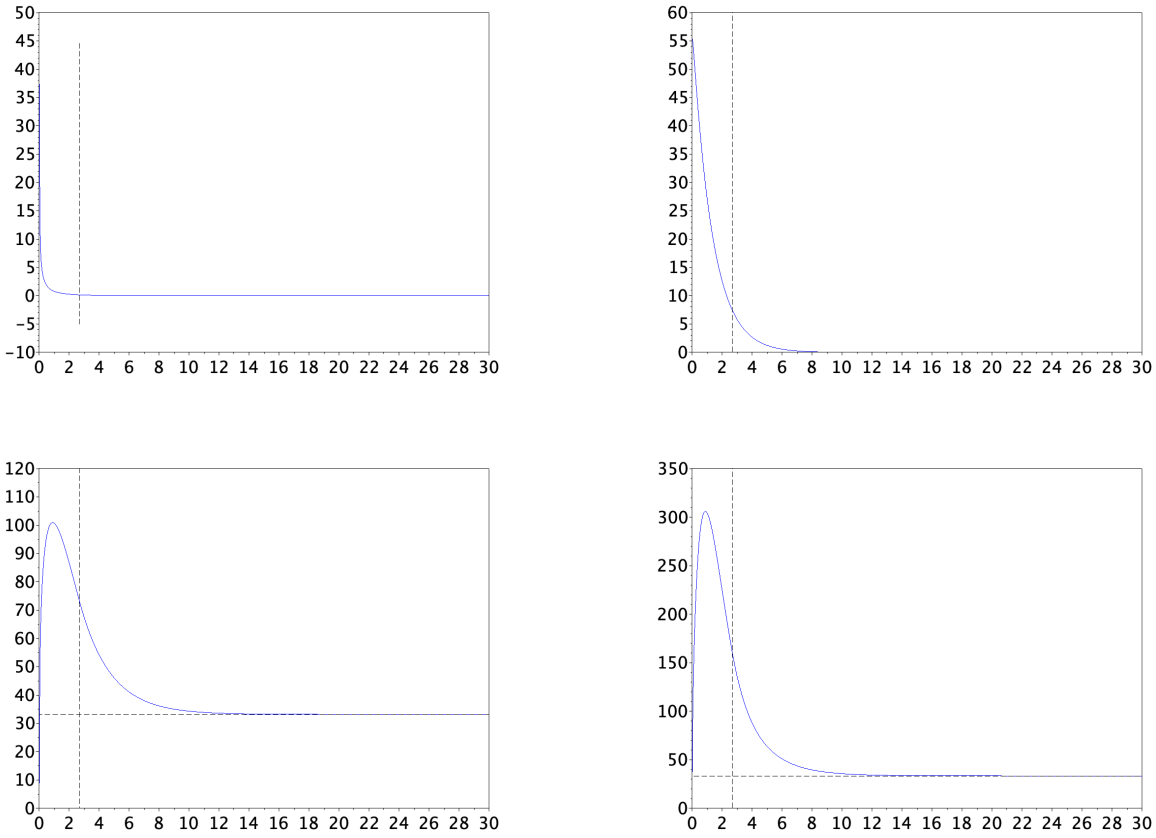

Figure 3: Release of adult mosquitoes, $k_{U}=1.01\left(\mathcal{R}_{W}-1\right)$. Evolution of the uninfecteds (top) and Wolbachia-infecteds (bottom), as functions of time. The larvae appear on the left column, the adults on the right one. The horizontal dotted lines show the asymptotic values at the complete infection equilibrium, the vertical dotted lines show the time instant after which the control input $u_{A}=\left|w^{\top} x\right|_{+}$is zero.
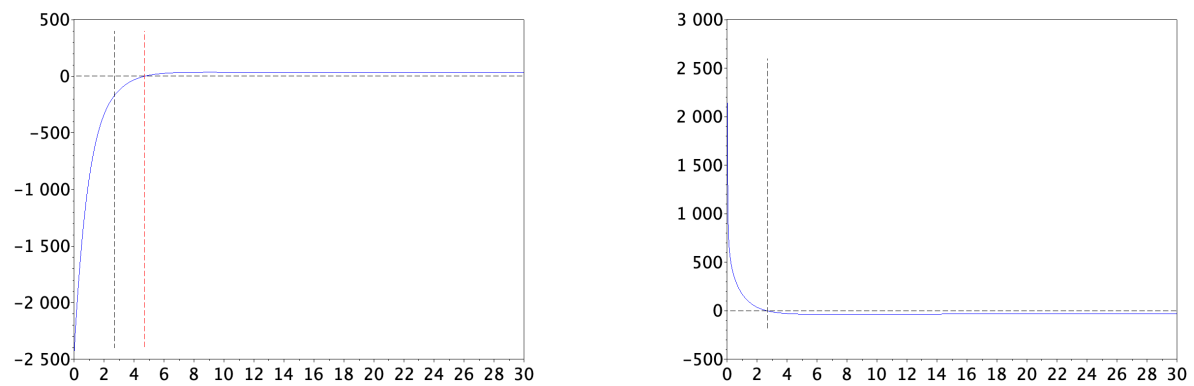

Figure 4: Release of adult mosquitoes, $k_{U}=1.01\left(\mathcal{R}_{W}-1\right)$. Evolution of the signals $A_{W}-k_{U} A_{U}$ (left) and $w^{\top} x$ (right) as functions of time. The black vertical dotted line shows the time instant after which $w^{\top} x$ is negative (and the control input $u_{A}$ is zero), the red vertical dotted line shows the time instant after which $A_{W}-k_{U} A_{U}>0$. 

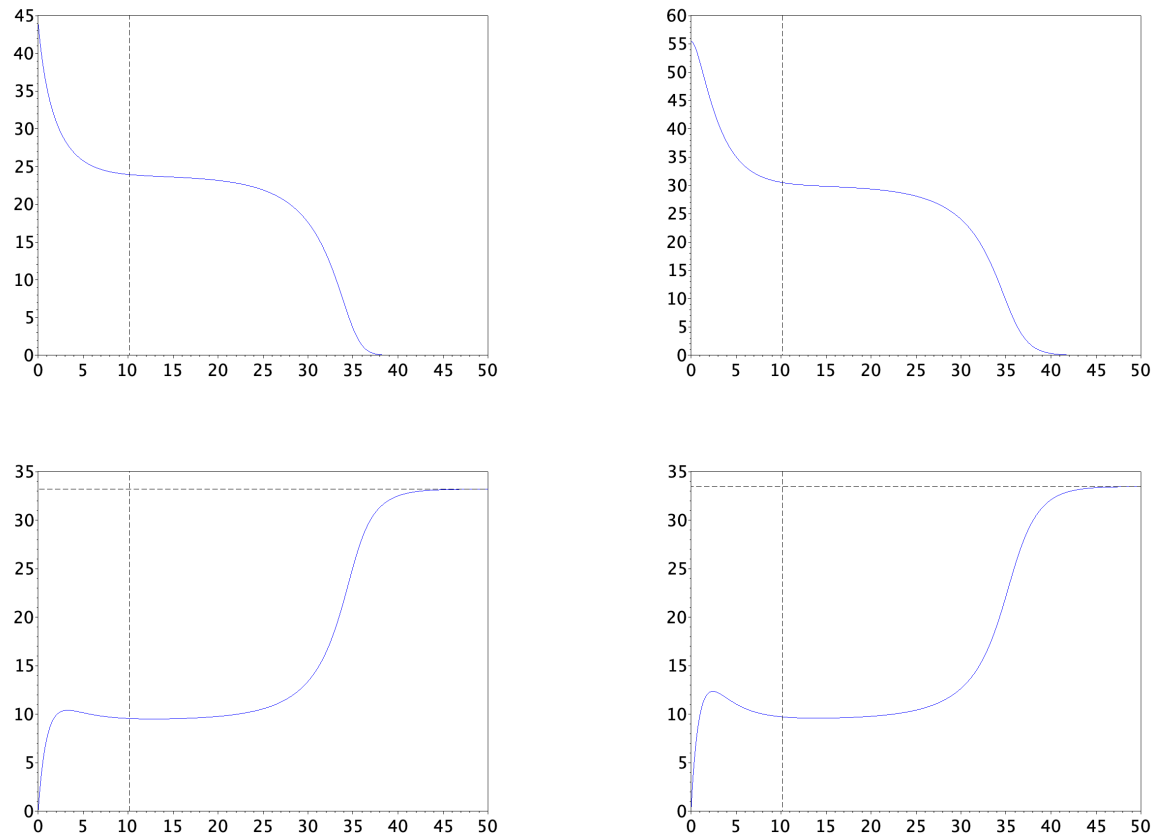

Figure 5: Release of adult mosquitoes, $k_{U}=1.01\left(\mathcal{R}_{W} / \mathcal{R}_{U}-1\right)$. Evolution of the uninfecteds (top) and Wolbachia-infecteds (bottom), as functions of time. The larvae appear on the left column, the adults on the right one. The horizontal dotted lines show the asymptotic values at the complete infection equilibrium, the vertical dotted lines show the time instant after which the control input $u_{A}=\left|w^{\top} x\right|_{+}$is zero.
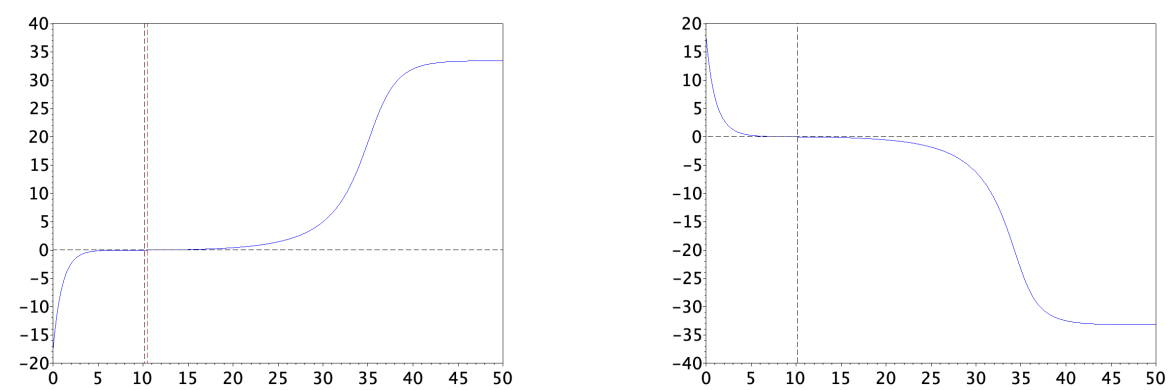

Figure 6: Release of adult mosquitoes, $k_{U}=1.01\left(\mathcal{R}_{W} / \mathcal{R}_{U}-1\right)$. Evolution of the signals $A_{W}-k_{U} A_{U}$ (left) and $w^{\top} x$ (right) as functions of time. The black vertical dotted line shows the time instant after which $w^{\top} x$ is negative (and the control input $u_{A}$ is zero), the red vertical dotted line shows the time instant after which $A_{W}-k_{U} A_{U}>0$. 


\subsection{Preliminary: constant releases}

We consider here the effect of the introduction of constant input $u$ in (1). In order to argue in all generality, we introduce a fixed proportion between the two components $u_{L}$ and $u_{A}$, and examine, for $\theta \in[0,1]$, the system (2) with the constant input

$$
u \equiv\left(\begin{array}{c}
\mathcal{R}_{W} \theta \\
1-\theta
\end{array}\right) v
$$

for fixed $v>0$. The case $\theta=1$, resp. $\theta=0$, corresponds to the introduction of constant rate of larvae, resp. adults.

Proposition 8 (Successful introduction by constant releases). For any $\theta \in$ $[0,1]$, there exists a unique real scalar $v_{\theta}^{*}>0$ such that the system

$$
\dot{x}=f(x)+B\left(\begin{array}{c}
\mathcal{R}_{W} \theta \\
1-\theta
\end{array}\right) v
$$

has two equilibria with positive $L_{U}, A_{U}$ if $0 \leqslant v<v_{\theta}^{*}$, and no positive equilibrium with positive $L_{U}, A_{U}$ if $v>v_{\theta}^{*}$. Moreover, in this latter case, (11) possesses a unique equilibrium. The latter displays complete infection and attracts every nonzero trajectory, and (6) is fulfilled for any initial condition.

Last, $v_{\theta}^{*}$ is defined as the smallest positive root of the 4-th order polynomial (in the variable $v$ )

$$
\Delta_{\theta}(v):=b^{2} c^{2}+18 a b c d-27 a^{2} d^{2}-4 a c^{3}-4 b^{3} d,
$$

where

$$
\begin{gathered}
a:=\mathcal{R}_{W}, \quad b:=b_{\theta}(v)=\mathcal{R}_{1}-\mathcal{R}_{2}+\frac{\gamma_{U}}{\gamma_{W}}(1-\theta) v \\
c:=c_{\theta}(v)=-\frac{\mathcal{R}_{1} \mathcal{R}_{2}}{\mathcal{R}_{W}}+\left(\frac{\gamma_{U}}{\gamma_{W}}(2-\theta)-1\right) v, \quad d:=d(v)=\left(\frac{\gamma_{U}}{\gamma_{W}}+\frac{\mathcal{R}_{2}}{\mathcal{R}_{W}}\right) v \\
\mathcal{R}_{1}:=\mathcal{R}_{W}-1, \quad \mathcal{R}_{2}:=\mathcal{R}_{U}-\mathcal{R}_{W}
\end{gathered}
$$

Proposition 8 presents no interest on its own, as constant releases necessitate permanent action, and therefore infinite cost (whatever the way it is computed), in contrast with the other results. It is provided as a preliminary to Theorem 9 ,

Proposition 8 is proved in Appendix A.6. Notice that, due to (3), $0<$ $\mathcal{R}_{1}<\mathcal{R}_{W}$ and $0<\mathcal{R}_{2}$. Also, remark that the existence of a smallest positive root for the polynomial $\Delta_{\theta}$ is guaranteed by the statement. 


\subsection{Saturated releases}

The following result establishes convergence for a saturated variant of the control law given in Theorem 5. Its proof, given in Appendix A.7, relies heavily on the fact that the corresponding controlled system with saturation is also monotone.

Theorem 9 (Saturated releases of larvae). Assume that, for some $T \geqslant 0$ and $u_{1}^{* *}>v_{1}^{*}$,

$$
u_{L}>\min \left\{u_{1}^{* *} ; L_{U} L_{W}\right\}, \quad u_{A} \geqslant 0 \quad \text { on }[T,+\infty) .
$$

Then all conclusions of Theorem 5 hold.

For the release of adults, the monotonicity argument does not hold, and the situation seems more complicated. In fact, we haven't succeeded in establishing convergence property for the following saturated version of $\mathbf{A}$ :

$$
u_{L} \geqslant 0, u_{A}>\min \left\{u_{0}^{* *} ;\left|w^{\top} x\right|_{+}\right\} \quad \text { on }[T,+\infty) .
$$

Nevertheless, associated simulations are also shown in the next Section.

\subsection{Numerical simulations - II}

4.3.1. Numerical determination of $v_{1}^{*}, v_{0}^{*}$

One first determines graphically the value of the first positive zero of the polynomials $\Delta_{0}(v)$ and $\Delta_{1}(v)$, as exposed in Proposition 8 , This yields:

$$
v_{0}^{*} \simeq 0.78226 \quad \text { and } \quad v_{1}^{*} \simeq 0.89387 .
$$

\subsubsection{Constant releases}

In order to check the previous values, we first simulate (11) for values of $v$ slightly subcritical (90\% of the threshold value $v_{\theta}^{*}$ ) and slightly supercritical $\left(110 \%\right.$ of the threshold value $\left.v_{\theta}^{*}\right)$. The results are shown in Figure 7 for larvae releases $(\theta=1)$ and in Figure 8 for adults releases. The equilibrium value $L_{W}^{*}, A_{W}^{*}$ at complete infestation for the uncontrolled system are shown in dashed lines.

The two figures are quite similar. In the subcritical cases, they show the establishment of a coexistence equilibrium (with the presence of infecteds and non-infecteds), while in the supercritical cases, the system converges towards an equilibrium state with only infected mosquitoes. Notice that in this latter case, the corresponding asymptotic values of $L_{W}, A_{W}$ are then strictly greater than the values $L_{W}^{*}, A_{W}^{*}$ at the complete infestation equilibrium for the uncontrolled system, due to the continued supply of mosquitoes. 

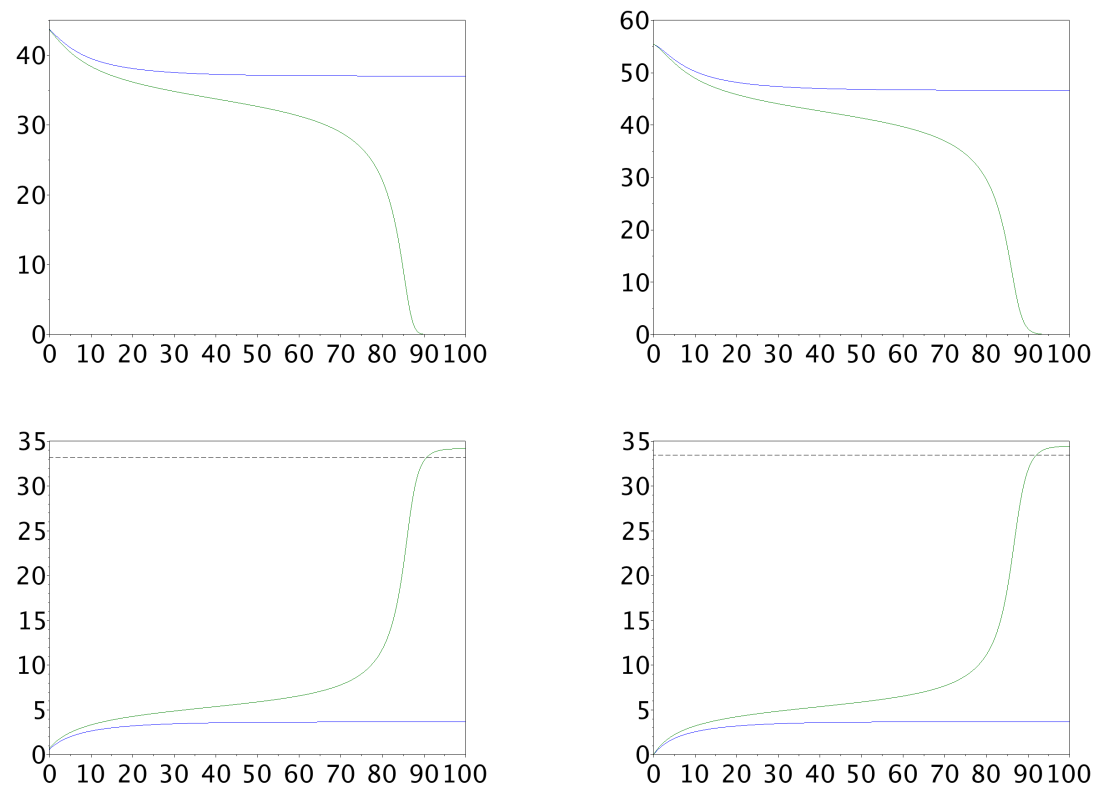

Figure 7: Release of larvae through constant control (Proposition 8, $\theta=1$ ): evolution of the uninfecteds (top) and Wolbachia-infecteds (bottom), as functions of time. The larvae appear on the left column, adults on the right one. The simulations show the cases $v=1.1 v_{1}^{*}$ (in green) and $v=0.9 v_{1}^{*}$ (in blue) in formula (11).
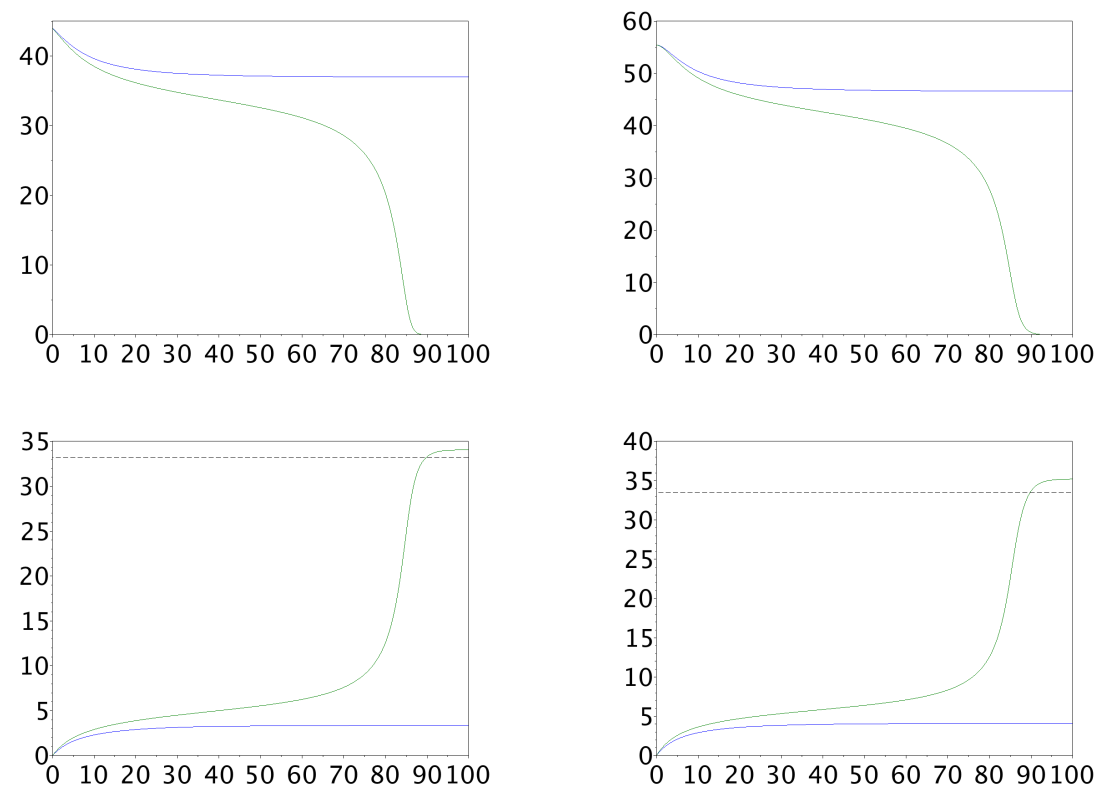

Figure 8: Release of adults through constant control (Proposition 8, $\theta=0$ ): evolution of the uninfecteds (top) and Wolb2Chia-infecteds (bottom), as functions of time. Larvae appear on the left column, adults on the right one. The simulations show the cases $v=1.1 \mathcal{R}_{W} u_{0}^{*}$ (in green) and $v=0.9 \mathcal{R}_{W} v_{0}^{*}$ (in blue) in formula (11). 


\subsubsection{Saturated releases of larvae}

Figures 9, 10, analogous to Figures 1, 2 in the non-saturated case, show time evolution of the populations and of the control input $u_{L}$ when equalities in $\left(\mathbf{L}^{\prime}\right)$ and saturation value $1.1 \mathcal{R}_{W} u_{0}^{*}$ are used to define the applied input. The establishment of the infection is about one order of magnitude longer with the saturated control. In the same time, the peak value of the input is also smaller, in approximately the same proportion, and the total number of released larvae is comparable in both situations, see Tables 1 and 3 .

\begin{tabular}{|l|l|}
\hline Total number of released larvae & $2.97 \cdot 10^{3}$ \\
\hline
\end{tabular}

Table 3: Number of released larvae until complete infection for the saturated control (see Section 4.3.3

\subsubsection{Saturated releases of adult mosquitos}

Figures 11 and 12 (resp. 13 and 14) are the analogues of Figures 3 and 4 (resp. 5 and 6), showing time evolution when control law (A') is applied with saturation value $1.1 u_{1}^{*}$ and with $k_{U}=1.01\left(\mathcal{R}_{W}-1\right)$ (resp. $\left.k_{U}=1.01\left(\mathcal{R}_{U} / \mathcal{R}_{W}-1\right)\right)$. The two saturated feedback laws yield comparable behaviours in terms of settling time and total number of released adults (see Table (4). As for the larvae release, the settling is slower with the saturated control law, but still more costly than the unsaturated one with the tuning $k_{U}=1.01\left(\mathcal{R}_{U} / \mathcal{R}_{W}-1\right)$.

\begin{tabular}{|l|l|}
\hline Total number of released adults, $k_{U}=1.01\left(\mathcal{R}_{U}-1\right)$ & $7.55 \cdot 10$ \\
\hline Total number of released adults, $k_{U}=1.01\left(\mathcal{R}_{U} / \mathcal{R}_{W}-1\right)$ & $6.61 \cdot 10$ \\
\hline
\end{tabular}

Table 4: Number of released adults until complete infection for the saturated control (see Section 4.3.4

\section{Upper and lower state estimates}

We provide here a class of interval observers for system (1). The relevance of this is that, based on these upper and lower estimates of the state variables, one may build directly output feedback control laws from Theorems 5, 6, 7 and 9, as they all specify lower bound to the input variables. Notice that 

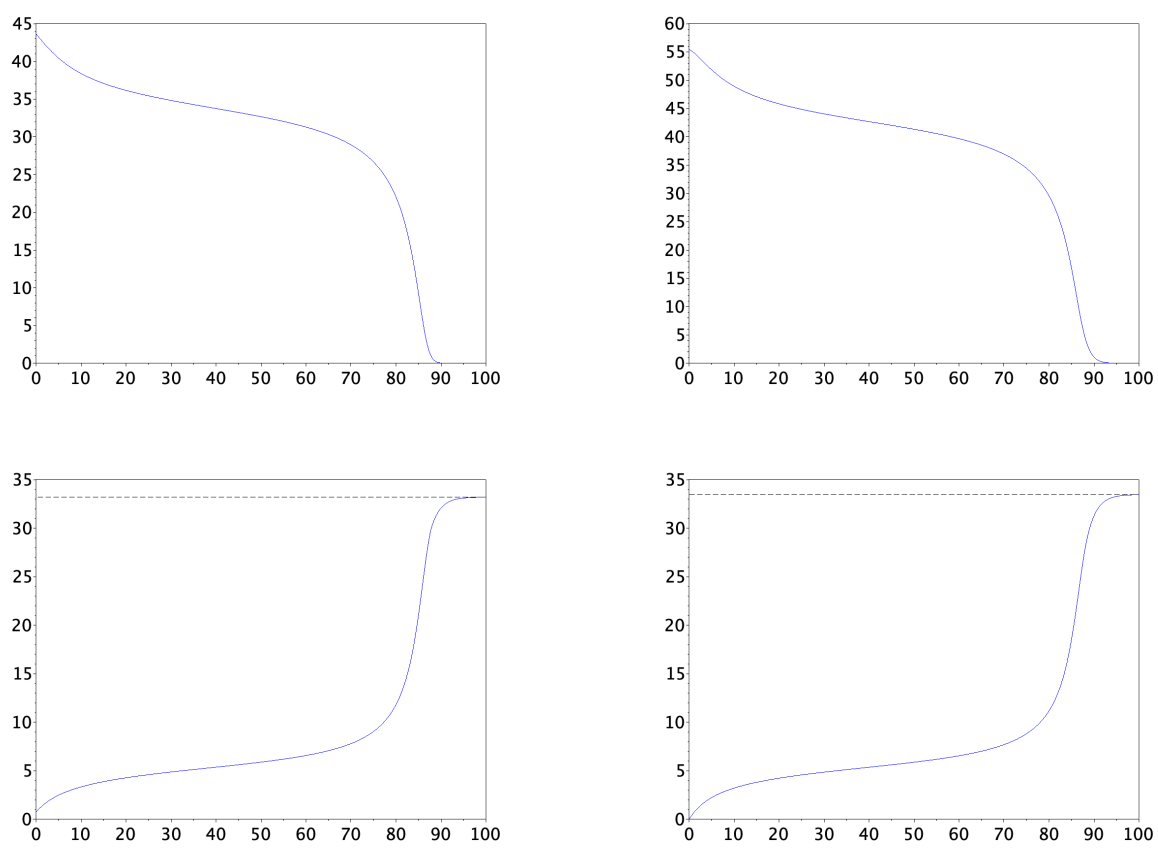

Figure 9: Release of larvae, with control saturation at $1.1 \mathcal{R}_{W} u_{0}^{*}$. Evolution of the uninfecteds (top) and Wolbachia-infecteds (bottom), as functions of time, with larvae on the left column and adults on the right one. The horizontal dotted lines show the asymptotic values at the complete infection equilibrium.

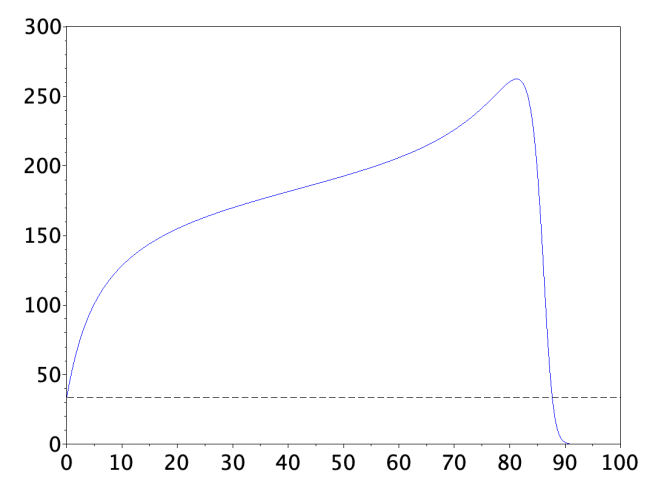

Figure 10: Release of larvae, with control saturation at $1.1 \mathcal{R}_{W} u_{0}^{*}$. Evolution of the control signal $L_{U} L_{W}$ as function of time. The saturation level is shown in dotted line, and the effective control input is $u_{L}=\min \left\{L_{U} L_{W} ; 1.1 \mathcal{R}_{W} u_{0}^{*}\right\}$. 

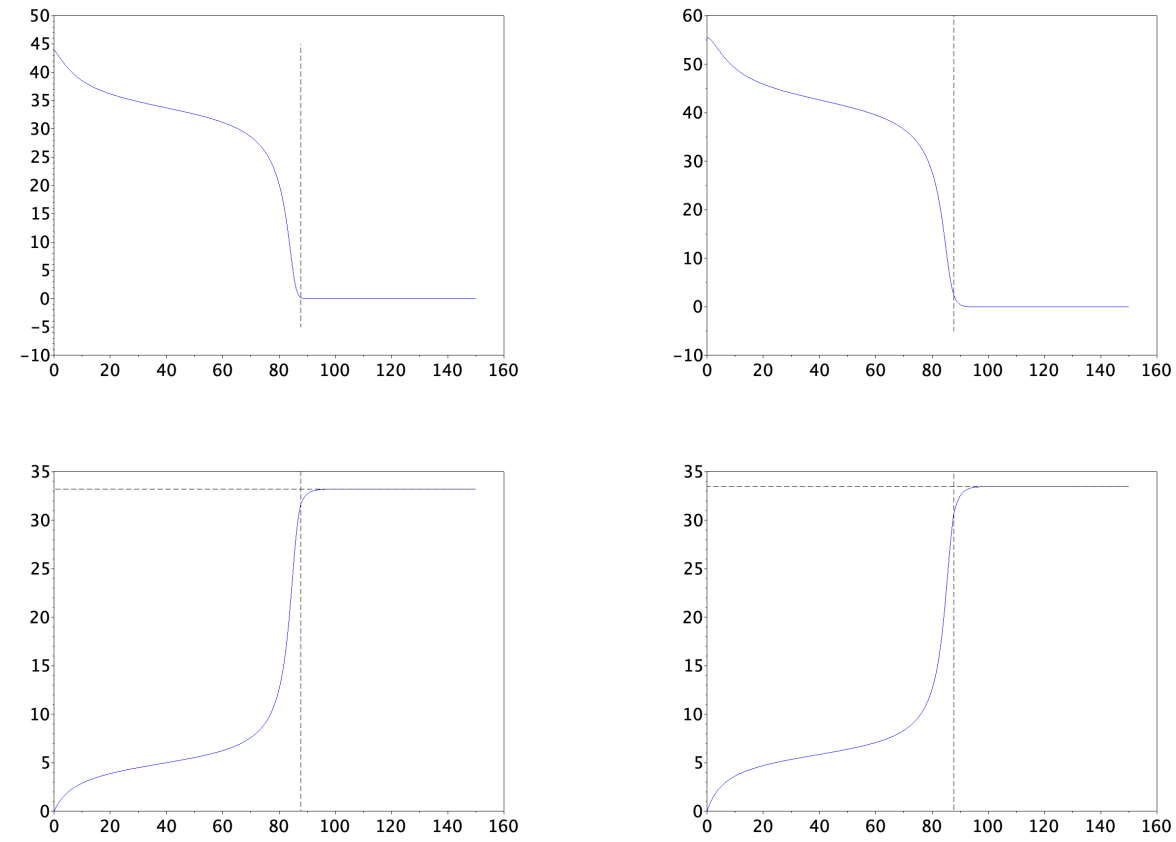

Figure 11: Release of adult mosquitoes, $k_{U}=1.01\left(\mathcal{R}_{W}-1\right)$, with control saturation at $1.1 v_{1}^{*} \simeq 0.8605$. Evolution of the uninfecteds (top) and Wolbachia-infecteds (bottom), as functions of time, with larvae on the left column and adults on the right one. For this value of $k_{U}$, the control signal $w^{\top} x$ crosses the saturation level $1.1 v_{1}^{*}$ immediately before reaching 0 , at a time instant shown by the black vertical dotted line.
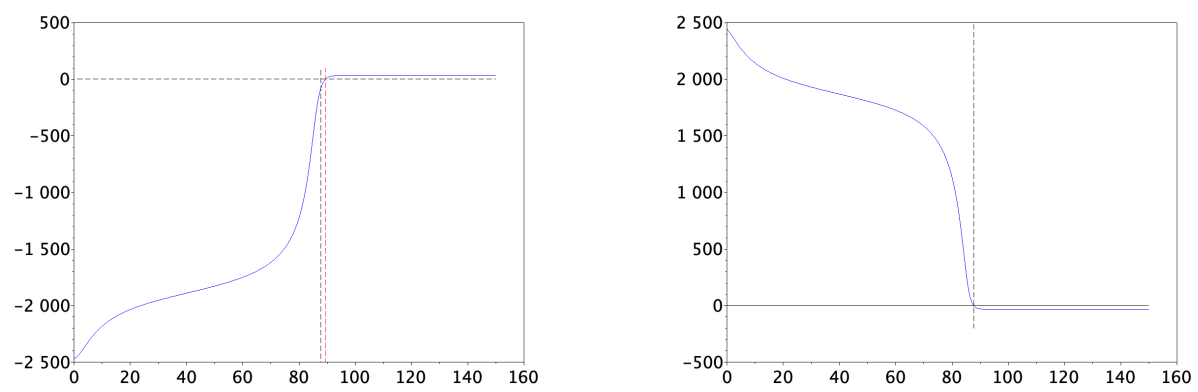

Figure 12: Release of adult mosquitoes, $k_{U}=1.01\left(\mathcal{R}_{W}-1\right)$, with control saturation at $1.1 v_{1}^{*} \simeq 0.8605$. Evolution of the signals $A_{W}-k_{U} A_{U}$ (left) and $w^{\top} x$ (right) as functions of time. The black vertical dotted line shows the time instant after which $w^{\top} x$ is negative and the control input $u_{A}=\min \left\{\left|w^{\top} x\right|_{+} ; 1.1 v_{1}^{*}\right\}$ is zero. The red vertical dotted line shows the time instant after which $A_{W}-k_{U} A_{U}>0$. 

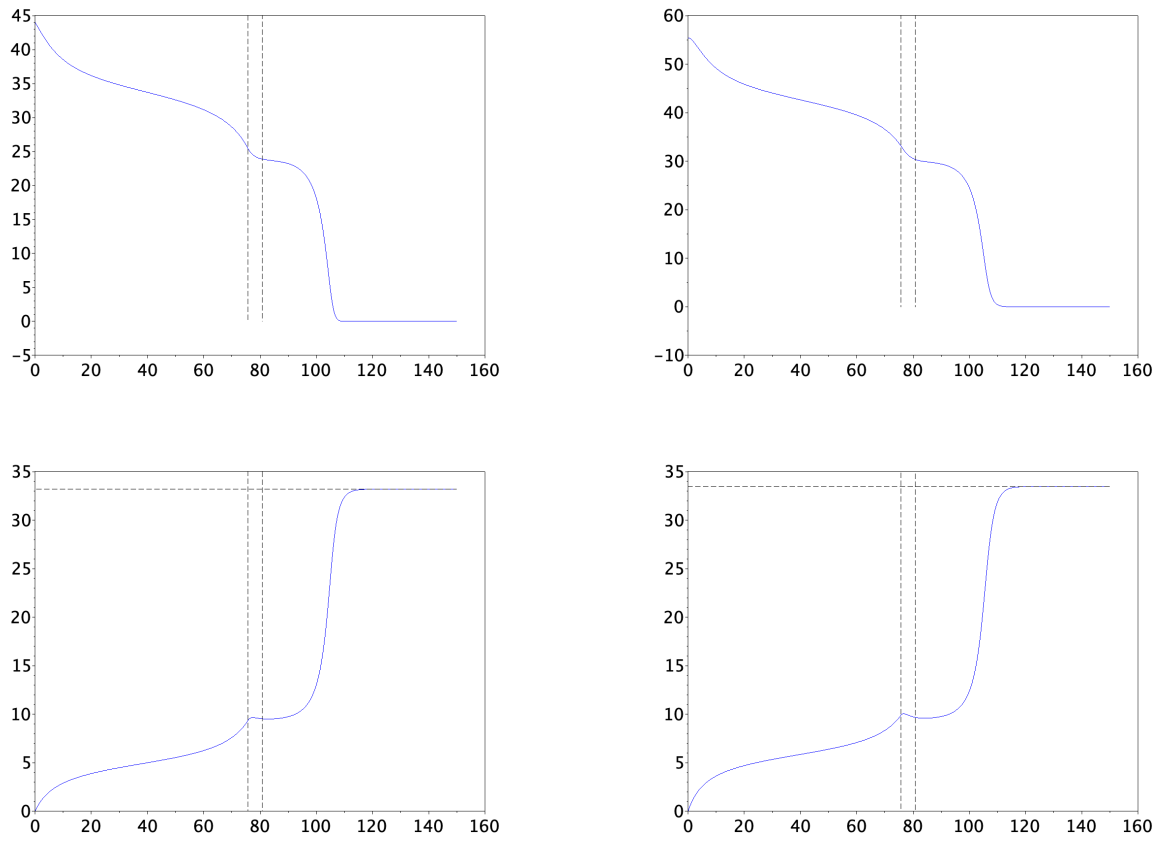

Figure 13: Release of adult mosquitoes, $k_{U}=1.01\left(\mathcal{R}_{W} / \mathcal{R}_{U}-1\right)$, with control saturation at $1.1 v_{1}^{*} \simeq 0.8605$. Evolution of the uninfecteds (top) and Wolbachiainfecteds (bottom), as functions of time, with larvae on the left column and adults on the right one. The control signal $w^{\top} x$ crosses successively the saturation level $1.1 v_{1}^{*}$ and 0 , at time instants shown by the black vertical dotted lines.
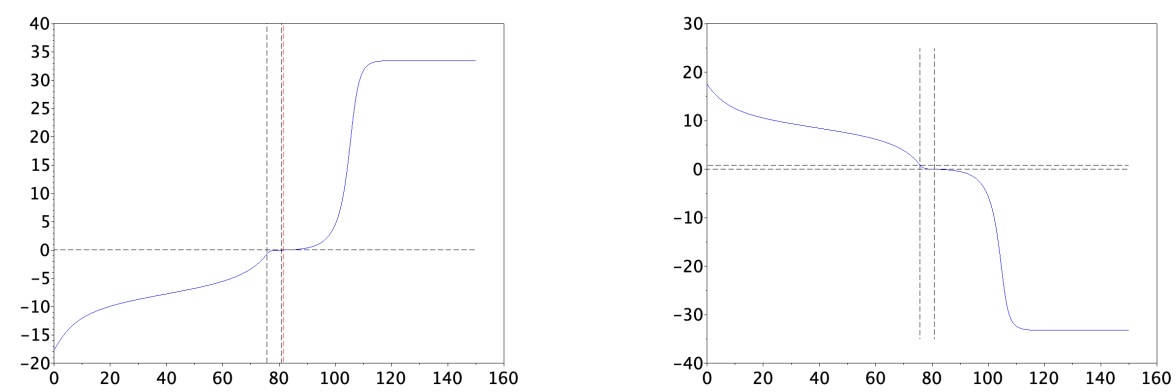

Figure 14: Release of adult mosquitoes, $k_{U}=1.01\left(\mathcal{R}_{W} / \mathcal{R}_{U}-1\right)$, with control saturation at $1.1 v_{1}^{*} \simeq 0.8605$. Evolution of the signals $A_{W}-k_{U} A_{U}$ (left) and $w^{\top} x$ (right) as functions of time. The black vertical dotted lines show the time instants at which $w^{\top} x$ crosses the saturation level $1.1 v_{1}^{*}$ and 0 (the control input is $u_{A}=\min \left\{\left|w^{\top} x\right|_{+} ; 1.1 v_{1}^{*}\right\}$ ). The red vertical dotted line shows the time instant after which $A_{W}-k_{U} A_{U}>0$. 
using Theorem 4, one has for any trajectory, regardless of the admissible input,

$$
\begin{aligned}
& 0 \leqslant \liminf _{t \rightarrow+\infty} L_{U}(t) \leqslant \limsup _{t \rightarrow+\infty} L_{U}(t) \leqslant L_{U}^{*}, \\
& 0 \leqslant \liminf _{t \rightarrow+\infty} A_{U}(t) \leqslant \limsup _{t \rightarrow+\infty} A_{U}(t) \leqslant A_{U}^{*} .
\end{aligned}
$$

The use of such rough estimates is possible, but may yield control laws uselessly costly: the present section presents more precise estimates.

We assume the availability of, say, $p$ measurements, and introduce the output variable $y=C x$ for some fixed matrix $C \in \mathbb{R}^{p \times 4}$. Typically $y$ contains measurement of the values of $L_{U}(t), L_{W}(t)$ and/or $A_{U}(t), A_{W}(t)$, depending upon the information monitored in the field.

In order to take into account measurement uncertainties, one assumes that are available two (locally integrable, nonnegative-valued) auxiliary signals $y_{-}(t), y_{+}(t)$ bounding the exact, but unknown, value $y(t)$ : for (almost) any $t \geqslant 0$

$$
0 \leqslant y_{-}(t) \leqslant y(t) \leqslant y_{+}(t)
$$

We then have the following result.

Theorem 10 (Interval observer design). For $x$ evolving according to (2), let the evolution of the variables

$$
x_{-}:=\left(\begin{array}{cccc}
L_{U}^{+} & A_{U}^{+} & L_{W}^{-} & A_{W}^{-}
\end{array}\right)^{\top}, \quad x_{+}:=\left(\begin{array}{llll}
L_{U}^{-} & A_{U}^{-} & L_{W}^{+} & A_{W}^{+}
\end{array}\right)^{\top}
$$

be defined by

$$
\begin{aligned}
& \dot{x}_{-}=f\left(x_{-}(t)\right)+B u+K_{-}\left(x_{-}(t)\right)\left(y_{-}(t)-C x_{-}(t)\right) \\
& \dot{x}_{+}=f\left(x_{+}(t)\right)+B u+K_{+}\left(x_{+}(t)\right)\left(y_{+}(t)-C x_{+}(t)\right)
\end{aligned}
$$

for gain matrices $K_{-}(\cdot), K_{+}(\cdot) \in \mathbb{R}^{4 \times p}$ depending continuously upon the state. Assume that

$$
\begin{gathered}
\operatorname{diag}\left\{-I_{2} ; I_{2}\right\} K_{ \pm}(\cdot) \geqslant 0 \\
\operatorname{diag}\left\{-I_{2} ; I_{2}\right\} K_{ \pm}(\cdot) C \operatorname{diag}\left\{-I_{2} ; I_{2}\right\} \leqslant 0
\end{gathered}
$$

and that the $i$-th line of $K_{-}(\cdot)$ (resp. $\left.K_{+}(\cdot)\right)$ is null whenever the $i$-th component of its argument is null, $i=3,4$ (resp. $i=1,2$ ).

If

$$
x_{-}(t) \leqslant_{C} x(t) \leqslant_{C} x_{+}(t) \text { and } 0 \leqslant x_{-}(t), x(t), x_{+}(t)
$$

for $t=0$, then the same holds true for any $t \geqslant 0$. 
Recall that $f$ in the previous statement is the function that allows to write (1) as (2). Theorem 10 provides sufficient conditions under which, if $0 \leqslant L_{\eta}^{-}(t) \leqslant L_{\eta}(t) \leqslant L_{\eta}^{+}(t), 0 \leqslant A_{\eta}^{-}(t) \leqslant A_{\eta}(t) \leqslant A_{\eta}^{+}(t), \eta=U, W$, for $t=0$, then the same holds true for any $t \geqslant 0$. In other words, system (13) constitutes an interval observer [27, 28, 29] for (1).

Coming back to the definition of the order $\leqslant_{C}$ in (5), conditions $114 \mathrm{a}$ (14b) appear as rephrasing of the conditions:

$$
\begin{aligned}
& \forall y, y^{\prime} \in \mathbb{R}^{p}, y \leqslant y^{\prime} \Rightarrow K_{-}(\cdot) y \leqslant_{C} K_{-}(\cdot) y^{\prime}, K_{+}(\cdot) y \leqslant_{C} K_{+}(\cdot) y^{\prime} \\
& \forall x, x^{\prime} \in \mathbb{R}^{4}, x \leqslant_{C} x^{\prime} \Rightarrow K_{-}(\cdot) C x \geqslant_{C} K_{-}(\cdot) C x^{\prime}, K_{+}(\cdot) C x \geqslant_{C} K_{+}(\cdot) C x^{\prime}
\end{aligned}
$$

For sake of space, we do not supply here numerical simulations involving these interval obersevers. More details and illustrations may be found in [32, 41].

Remark 3. Notice that $(14 \mathrm{~b}$ comes as a consequence of $114 \mathrm{a}$ when e.g.

$$
C \operatorname{diag}\left\{-I_{2} ; I_{2}\right\} \leqslant 0 \text {. }
$$

When for example measurements of the two larval populations $L_{U}(t), L_{W}(t)$ are available, then $p=2$ and one may take $C=\left(\begin{array}{cccc}1 & 0 & 0 & 0 \\ 0 & 0 & -1 & 0\end{array}\right)$, in accordance with (14c). According to (14a), the first two rows of the admissible gain matrices $K_{-}(\cdot), K_{+}(\cdot)$ must then be nonpositive, and their last two rows nonnegative.

Remark 4. A simple way to fulfill the last technical assumption on the matrix gain lines is to introduce a smoothed Heaviside function for the corresponding components; namely to take the $i$-th line of $K_{ \pm}(x)$ equal to the $i$-th line of fixed matrix $M_{ \pm}$fulfilling (16), multiplied by the function $\max \left\{\min \left\{x_{i}, \varepsilon\right\}, 0\right\}$, for some small $0<\varepsilon$. As an example, taking these matrices positively proportional to

$$
\left(\begin{array}{llll}
-1 & -1 & 1 & 1 \\
-1 & -1 & 1 & 1
\end{array}\right)^{\top}
$$

is convenient for $C$ defined in Remark 3 .

Remark 5. Techniques identical to the ones used in the proof of Theorem 10 permit to show that, if in addition to the assumptions therein, the first (resp. 
second) line of $K_{-}(\cdot)$ is null whenever the first (resp. second) component of its argument is equal to $L_{U}^{*}$ (resp. $\left.A_{U}^{*}\right)$, then

$$
x_{-}(t) \leqslant_{C} x(t) \leqslant_{C} x_{+}(t) \text { and } x_{-}(t), x(t), x_{+}(t) \in \mathcal{S}
$$

at $t=0$ implies the same property for any $t \geqslant 0$.

Proof of Theorem 10. For simplicity, we omit in the sequel the argument of the matrix-valued functions $K_{ \pm}$.

When $x_{-} \leqslant_{C} x$ and $y_{-} \leqslant y=C x$, one has, using (16): $K_{-}\left(y_{-}-C x_{-}\right)=$ $K_{-}\left(y_{-}-y\right)+K_{-} C\left(x-x_{-}\right) \leqslant_{C} 0$; and similarly, when $x_{+} \geqslant_{C} x$ and $y_{+} \geqslant y$ : $K_{+}\left(y_{+}-C x_{+}\right)=K_{+}\left(y_{+}-y\right)+K_{+} C\left(x-x_{+}\right) \geqslant_{C} 0$. Therefore, for the system (2)-(13) one has $\dot{x}_{-} \leqslant_{C} f\left(x_{-}(t)\right), \dot{x}_{+} \geqslant_{C} f\left(x_{+}(t)\right)$.

Use of Kamke's theorem [44] allows to show that when the 1st property in (15) holds for $t=0$, it holds also for any $t \geqslant 0$, provided that all components of the three vectors $x, x_{-}, x_{+}$remain nonnegative - otherwise the composition by $f$ is not licit, and the solution is not defined.

The variable $x$, whose evolution does not depend upon $x_{-}, x_{+}$, remains nonnegative along time. We now show the same properties for $x_{-}, x_{+}$. First, one verifies easily that the indexes mentioned in the statement are exactly those related to the evolution of $L_{U}^{-}, A_{U}^{-}, L_{W}^{-}, A_{W}^{-}$. On the other hand, when $\left(x_{ \pm}\right)_{i}=0$ for some $i=1, \ldots, 4$, the $i$-th component $\left(f\left(x_{ \pm}\right)\right)_{i}$ is nonnegative. Thus, for any matrix line concerned by the condition imposed in the statement, $\left(\dot{x}_{ \pm}\right)_{i} \geqslant 0$ whenever $\left(x_{ \pm}\right)_{i}=0$, and for this reason the values of the corresponding signals never leave the interval $[0,+\infty)$. With an initialization done in accordance with (15), the lower estimates are thus nonnegative, which forces in turn the components $L_{U}^{+}, A_{U}^{+}, L_{W}^{+}, A_{W}^{+}$to be nonnegative. Therefore the 2nd inequality in (15) also holds, and this achieves the proof of Theorem 10.

\section{Conclusion}

Several release strategies have been proposed, allowing to realize complete infestation of a population of Aedes mosquitoes by a population artificially infected by the bacterium Wolbachia, which reduces their ability as vectors of several important diseases. More specifically, the goal of the paper is to provide three 'general control principles' upon which to build feedback control laws in various situations, namely: to compensate for the competition terms that restrict the growth of the infected population due to the presence of the 
uninfected; to constrain the uninfected population to have subcritical fitness; and to constraint the uninfected population to have fitness smaller than the infected population fitness. We deduced from these principles several control laws, whose efficiency has been demonstrated analytically and tested numerically. While the main results are presented in terms of state-feedback control laws, interval observers are also provided that allow to design easily output-state feedback control laws. Saturated control has been studied as a complement, enabling reduction of the peak value of the control input, but showing small room for diminution of the total number of released insects through reduction of the release pace.

The main assets of the presented results are their generality and the fact that they are based on the monotonicity of the model. This last aspect is believed to be a good way to obtain infection strategies robust against the unmodeled dynamics and parameter uncertainties that are unavoidable in such biological application, with no need of a precise knowledge of the boundary between the basins of attraction of the infective and non-infective equilibria. One may check that most of the models of Wolbachia spread cited in the introduction are monotone, and this mathematical property certainly reflects solid traits of the contemplated experimental situation.

From a technical point of view, loss of monotonicity may occur as an undesirable consequence of a more precise description of the phenomena involved: incomplete vertical transmission, development delays (as well as measurement or actuation delays in the control loop), sex- or age-structure introduce mutual influences that frustrate the cooperative or competitive nature of the interactions between the two populations as it is captured in the coarse model used in this paper. Extension of the arguments to non-monotone systems is certainly one of the next important points to be considered, and the comparison results abundantly used in the present paper, which allow to compare trajectories of two related systems provided at least one is monotone, constitute undoubtedly a tempting tool to tackle this question. Notice that monotonicity may also be a powerful starting point to deal with more realistic release setup, such as impulsive and periodic releases, much in the spirit of [45] in the context of Sterile Insect Techniques.

Last, we would like to stress the potential interest of the contribution presented here to tackle other important issues related to the control of vectors and pests. It is definitely our belief that after the great steps made by J.-L. Gouzé [46], D. Angeli and E. Sontag [40, 47] and subsequent researchers [48, 49, 50], Monotone systems theory has not yet revealed all its 
powerfulness in the field of Control theory.

\section{Acknowledgments}

The author thanks the anonymous reviewers for their careful reading, which allowed significant improvement of the manuscript. He acknowledges the support of the Franco-Columbian program ECOS-Nord (project C17M01); of the program STIC AmSud (project 20-STIC-05); and of Inria through the Associate Team MOCOVEC. This work also benefited from fruitful discussions with Yves Dumont (CIRAD, University of Pretoria) within the framework of the Phase 2B 'SIT feasibility project against Aedes albopictus in Reunion Island', jointly funded by the French Ministry of Health and the European Regional Development Fund (ERDF).

\section{Appendix A. Proofs}

\section{Appendix A.1. Proof of Theorem 1}

The nonnegativeness of the solution comes from the fact that $\dot{L} \geqslant 0$ (resp. $\dot{A} \geqslant 0$ ) whenever $L=0$ (resp. $A=0$ ). The values of the equilibrium points are computed directly.

Using Kamke's condition, it is straightforward to show that system (4) is cooperative and irreducible [31, Chapter 4], and therefore SOP.

- In the case where $\mathcal{R} \leqslant 1, x_{0}^{*}$ is the unique equilibrium, and every point of $\mathbb{R}_{+}^{2} \backslash\left\{x_{0}^{*}\right\}$ may be approximated from below and from above [31, p. 8] in this set. The global asymptotic stability of $x_{0}^{*}$ is then deduced from [31, Chapter 2, Theorem 3.1, p. 18].

- We now treat the case where $\mathcal{R}>1$. Notice that the two equilibrium points are ordered: $x_{0}^{*} \ll x^{*}$. Therefore the order interval

$$
\llbracket x_{0}^{*} ; x^{*} \rrbracket:=\left\{(L, A): 0 \leqslant L \leqslant L^{*}, 0 \leqslant A \leqslant A^{*}\right\}
$$

is non empty, compact and forward invariant. As there is no other equilibrium point in the corresponding order interval, one may apply a trichotomy result [31, Chapter 2, Theorem 2.2, p. 17] to show that one of the two equilibrium points attracts every trajectory beginning within this interval (except of course the other equilibrium). One shows directly that the Jacobian matrix of the system at $x_{0}^{*}$ is unstable, while it is Hurwitz at $x^{*}$ : the trajectory departing from any point in $\llbracket x_{0}^{*} ; x^{*} \rrbracket \backslash\left\{x_{0}^{*}\right\}$ thus converges towards $x^{*}$. 
To close, consider an initial point $x:=(L, A)$ outside the order interval $\llbracket x_{0}^{*} ; x^{*} \rrbracket$. Then, construct the point $x^{\prime}:=\left(L^{\prime}, A^{\prime}\right)$ as

$$
L^{\prime}:=\max \{L, \gamma A\}, \quad A^{\prime}:=\frac{1}{\gamma} L^{\prime}
$$

One shows directly that $x_{0}^{*} \leqslant x \leqslant x^{\prime}$ for the product order in $\mathbb{R}^{2}$, and that $x^{*} \leqslant x^{\prime}$. By construction, the derivative of the flow at the point $x^{\prime}$ is nonpositive. Therefore, the trajectory originated from this point is nonincreasing [31, Chapter 3, Proposition 2.1, p. 34]. Being bounded from below by the equilibrium $x^{*}$, it thus converges, and its limit is necessarily the equilibrium $x^{*}$, because $x^{\prime} \geqslant x^{*}$. As $x \leqslant x^{\prime}$, the trajectory coming from the former point is dominated by the one coming from the latter one, and its $\omega$-limit set is thus within the order interval $\llbracket x_{0}^{*} ; x^{*} \rrbracket$. Considering the previous case, one deduces that in this case too the trajectory converges to $x^{*}$. This achieves the proof of Theorem 1 .

\section{Appendix A.2. Proof of Theorem 2}

Most of the results in the statement have been originally demonstrated in [16], the reader is referred to this source for more details. The non negativeness and boundedness properties may be proved with arguments similar to the ones used in Appendix A.1 for system (4), see also [16, Theorem 1]. Finding the explicit value of the equilibria is straightforward, as well as proving their ordering [16, Theorem 7]. The local asymptotic stability of the two homogeneous equilibria is proved by checking that the Jacobian matrix is Hurwitz at these points; and the fact that the extinction equilibrium is repelling comes from the existence of a vector $v \in \mathbb{R}_{+}^{4}, v \gg 0$, such that the function $v^{\top} x(t)$ increases along the trajectories of the system in any pointed neighbourhood of this equilibrium in $\mathbb{R}_{+}^{4}$ [16, Theorem 7]. The instability of the coexistence equilibrium $x_{U W}^{*}$ is proved by applying to the interval $\llbracket x_{U W}^{*} ; x_{W}^{*} \rrbracket$ the same argument, adapted from [31, Chapter 2, Theorem 2.2, p. 17], that is used in the proof of Theorem 1. Last, the property on the union of the basins of attraction comes as a consequence of the existence of the coexistence equilibrium and the ejective nature of the extinction equilibrium $x_{0}^{*}$, through application of [39, Theorem 3.2]. 
Appendix A.3. Proof of Theorem 5

- 1. When $(\mathbf{L})$ is fulfilled, then the infected population obeys the following differential inequalities:

$$
\dot{L}_{W}>\gamma_{W} \mathcal{R}_{W} A_{W}-\left(1+L_{W}\right) L_{W}, \quad \dot{A}_{W} \geqslant L_{W}-\gamma_{W} A_{W}
$$

One may thus bound from below each of its solutions by a solution $(L, A)$ of system (4), with $\gamma:=\gamma_{W}$ and $\mathcal{R}:=\mathcal{R}_{W}$, initialized with the same value than $\left(L_{W}, A_{W}\right)$ in any $T^{\prime} \geqslant T$. As $\mathcal{R}_{W}>1$ (due to (3)), this solution of (4) tends to the positive equilibrium $x_{W}^{*}$, unless the initial condition is precisely the null extinction equilibrium.

- 2. We now use the 1 st inequality in $(\mathbf{L})$. Due to its strict character ${ }^{4}$ any trajectory of (1) takes on some nonzero value on $[T,+\infty)$, say at time $T^{\prime}$. As $(0,0)<\left(L\left(T^{\prime}\right), A\left(T^{\prime}\right)\right)$, convergence of $(L, A)$ to the zero equilibrium of (4) is excluded, and the solution is forced to converge towards the positive equilibrium. Using this property one deduces that the 2 nd inequality in (6) is fulfilled, with equality if $u_{L}, u_{A}$ vanish at infinity.

- 3. In turn, we deduce from the preceding inference, that the evolution of the non-infected population fulfills asymptotically the inequalities:

$$
\dot{L}_{U} \leqslant \gamma_{U} \mathcal{R}_{U} \frac{A_{U}}{A_{U}+A_{W}^{*}} A_{U}-\left(1+L_{W}^{*}+L_{U}\right) L_{U}, \quad \dot{A}_{U}=L_{U}-\gamma_{U} A_{U}
$$

The underlying differential system

$$
\dot{L}=\gamma_{U} \mathcal{R}_{U} \frac{A}{A+A_{W}^{*}} A-\left(1+L_{W}^{*}+L\right) L, \quad \dot{A}=L-\gamma_{U} A
$$

behaves qualitatively as (4): it is monotone (for the usual ordering induced by the orthant $\left.\mathbb{R}_{+}^{2}\right)$, admits only bounded trajectories and possesses $(0,0)$ as unique equilibrium (otherwise, a supplementary equilibrium would exist for the initial system (11)). Therefore arguing as in the proof of Theorem 1 (case $\mathcal{R} \leqslant 1$ ) with the help of [31, Chapter 2, Theorem 3.1, p. 18], every trajectory of system A.2 converges towards the unique equilibrium $(0,0)$. The system of differential inequalities A.1 has nonnegative solutions that are at most equal to those of $(\mathrm{A} .2)$. One thus infers the 1st inequality of (6), and the proof of Theorem 5 is completed.

\footnotetext{
${ }^{4}$ The use of $>$ in this context is defined in the Notations paragraph, see Section 1.
} 
Appendix A.4. Proof of Theorem 6

- 1. First, we have

$$
\dot{A}_{W}-k_{U} \dot{A}_{U}=L_{W}-\gamma_{W} A_{W}+u_{A}-k_{U}\left(L_{U}-\gamma_{U} A_{U}\right)
$$

while by assumption $(\mathbf{A}$, one has on $[T,+\infty)$ :

$$
u_{A}>k_{U} L_{U}+k_{U}\left(k-\gamma_{U}\right) A_{U}-L_{W}+\left(\gamma_{W}-k\right) A_{W}
$$

By substitution, one deduces the 1st-order inequality (9). Integrating the latter yields, for any $t \geqslant T$,

$$
A_{W}(t)-k_{U} A_{U}(t) \geqslant e^{-k(t-T)}\left(A_{W}(T)-k_{U} A_{U}(T)\right) .
$$

Thus, as $k>0$,

$$
\liminf _{t \rightarrow+\infty}\left(A_{W}(t)-k_{U} A_{U}(t)\right) \geqslant 0 .
$$

- 2. As $k_{U}>\mathcal{R}_{U}-1$, there exists $\varepsilon>0$ such that $k_{U}>\mathcal{R}_{U}(1+\varepsilon)-1$. Due to A.3), there exists $T^{\prime} \geqslant T$ such that, for any $t \geqslant T^{\prime}, A_{W}(t) \geqslant$ $\left(\mathcal{R}_{U}(1+\varepsilon)-1\right) A_{U}(t)$. Then, due to the fact that the function $\mathbb{R}_{+} \rightarrow \mathbb{R}_{+}$, $z \mapsto \frac{1}{1+z}$ is decreasing,

$$
\mathcal{R}_{U} \frac{A_{U}(t)}{A_{U}(t)+A_{W}(t)} \leqslant \frac{\mathcal{R}_{U}}{1+\mathcal{R}_{U}(1+\varepsilon)-1}=\frac{1}{1+\varepsilon} .
$$

For $t \geqslant T^{\prime}$, the evolution of the unifected thus obeys the following inequalities:

$\dot{L}_{U} \leqslant \frac{\gamma_{U}}{1+\varepsilon} A_{U}-\left(1+L_{W}+L_{U}\right) L_{U} \leqslant \frac{\gamma_{U}}{1+\varepsilon} A_{U}-\left(1+L_{U}\right) L_{U}, \quad \dot{A}_{U}=L_{U}-\gamma_{U} A_{U}$

System (4) with $\gamma:=\gamma_{U}, \mathcal{R}:=\frac{1}{1+\varepsilon}<1$ may serve as a comparison system: denoting $(L, A)$ the solution of (4) such that $L\left(T^{\prime}\right)=L_{U}\left(T^{\prime}\right), A\left(T^{\prime}\right)=$ $A_{U}\left(T^{\prime}\right)$, one has, for any $t \geqslant T^{\prime}$,

$$
0 \leqslant L_{U}(t) \leqslant L(t), \quad 0 \leqslant A_{U}(t) \leqslant A(t)
$$

On the other hand, as $\mathcal{R}<1,(L(t), A(t))$ vanishes when $t \rightarrow+\infty$. One thus gets:

$$
\lim _{t \rightarrow+\infty} L_{U}(t)=\lim _{t \rightarrow+\infty} A_{U}(t)=0
$$


which is the 1st inequality of (6).

- 3. We now deduce lower bounds on $L_{W}, A_{W}$ from the identities in (A.4). For any $\varepsilon>0$, there exists $T^{\prime \prime}>T$ such that, for any $t \geqslant T^{\prime \prime}, 0 \leqslant L_{U}(t) \leqslant \varepsilon$. Using the fact that $u_{A}, u_{L}$ have nonnegative values, one gets:

$$
\dot{L}_{W} \geqslant \gamma_{W} \mathcal{R}_{W} A_{W}-\left(1+\varepsilon+L_{W}\right) L_{W}, \quad \dot{A}_{W} \geqslant L_{W}-\gamma_{W} A_{W} .
$$

The underlying system

$$
\dot{L}^{\prime}=\gamma_{W} \mathcal{R}_{W} A^{\prime}-\left(1+\varepsilon+L^{\prime}\right) L^{\prime}, \quad \dot{A}^{\prime}=L^{\prime}-\gamma_{W} A^{\prime}
$$

leads by normalization to (4), with $\mathcal{R}=\frac{\mathcal{R}_{W}}{1+\varepsilon}$. Applying again comparison result [44], one gets that, for any $t \geqslant T^{\prime \prime}$,

$$
L_{W}(t) \geqslant L^{\prime}(t), \quad A_{W}(t) \geqslant A^{\prime}(t)
$$

for the trajectory of A.5 initialized by $L^{\prime}\left(T^{\prime \prime}\right)=L_{W}\left(T^{\prime \prime}\right), A^{\prime}\left(T^{\prime \prime}\right)=A_{W}\left(T^{\prime \prime}\right)$.

For $0<\varepsilon<\mathcal{R}_{W}-1$, every nonzero trajectory of A.5 converges towards the positive equilibrium $\left(\mathcal{R}_{W}-1-\varepsilon, \frac{\mathcal{R}_{W}-1-\varepsilon}{\gamma_{W}}\right)$. Thus,

$$
\lim _{t \rightarrow+\infty} L^{\prime}(t)=\mathcal{R}_{W}-1-\varepsilon, \quad \lim _{t \rightarrow+\infty} A^{\prime}(t)=\frac{\mathcal{R}_{W}-1-\varepsilon}{\gamma_{W}} .
$$

Deducing from (A.6) that

$$
\begin{aligned}
& \liminf _{t \rightarrow+\infty} L_{W}(t) \geqslant \lim _{t \rightarrow+\infty} L^{\prime}(t)=\mathcal{R}_{W}-1-\varepsilon, \\
& \liminf _{t \rightarrow+\infty} A_{W}(t) \geqslant \lim _{t \rightarrow+\infty} A^{\prime}(t)=\frac{\mathcal{R}_{W}-1-\varepsilon}{\gamma_{W}},
\end{aligned}
$$

and passing to the limit $\varepsilon \rightarrow 0$, yields the 2nd inequality in (6). When $u_{L}, u_{A}$ vanish at infinity, the same comparison techniques permit to bound the trajectories from above and obtain equality. This completes the proof of Theorem 6 .

Appendix A.5. Proof of Theorem 7

Arguing as for Theorem 6, one shows that A.3 holds, with here $k_{U}>$ $\mathcal{R}_{U} / \mathcal{R}_{W}-1$. For any $\varepsilon>0$ for which $k_{U}>\frac{\mathcal{R}_{U}}{\mathcal{R}_{W}}(1+\varepsilon)-1$, consider $T^{\prime} \geqslant T$ such that, for any $t \geqslant T^{\prime}$,

$$
\mathcal{R}_{U} \frac{A_{U}(t)}{A_{U}(t)+A_{W}(t)}<\frac{\mathcal{R}_{W}}{1+\varepsilon} .
$$


For any $t \geqslant T^{\prime}$, the evolution of the system along the studied trajectory fulfils

$$
\begin{gathered}
\dot{L}_{U} \leqslant \gamma_{U} \frac{\mathcal{R}_{W}}{1+\varepsilon} \frac{A_{U}}{A_{U}+A_{W}} A_{U}-\left(1+L_{U}+L_{W}\right) L_{U} \\
\dot{A}_{U}=L_{U}-\gamma_{U} A_{U} \\
\dot{L}_{W} \geqslant \gamma_{W} \mathcal{R}_{W} A_{W}-\left(1+L_{U}+L_{W}\right) L_{W} \\
\dot{A}_{W} \geqslant L_{W}-\gamma_{W} A_{W}
\end{gathered}
$$

Notice that the previous set of inequalities define an inequality in the $\geqslant_{C}$ sense. The differential system underlying (A.7) is quite similar to (1), and in particular it is SOP. Computations that present no difficulties demonstrate that, due to the fact that $\varepsilon>0$, it possesses three equilibrium points, namely the extinction equilibrium and the two homogeneous equilibria $x_{W}^{*}$ (infected) and

$$
x_{U}^{\prime *}:=\left(\begin{array}{llll}
\frac{\mathcal{R}_{W}}{1+\varepsilon}-1 & \frac{1}{\gamma_{U}}\left(\frac{\mathcal{R}_{W}}{1+\varepsilon}-1\right) & 0 & 0
\end{array}\right)^{\top}
$$

(noninfected). The extinction equilibrium is repelling, as is the case for (1), and direct computations that exploit the block-diagonal structure of the Jacobian matrix at these points show that $x_{W}^{*}$ is LAS while $x_{U}^{\prime *}$ is unstable.

On the other hand, the system is in the configuration of competition between two agents described in [39, Section 3]. In absence of a coexistence equilibrium, one is allowed to deduce from [39, Theorem 3.1] that all its trajectories departing from $\llbracket x_{U}^{*}, x_{W}^{*} \rrbracket$ converges towards $x_{W}^{*}$. One concludes the proof of Theorem 7 with the help of comparison result.

\section{Appendix A.6. Proof of Proposition 8}

The proof is organized as follows. One first shows the existence of the threshold value $v_{\theta}^{*}$, then provides its characterization by mean of the polynomial $\Delta_{\theta}$ defined in (12), and finally studies the asymptotic behavior of system (11) when $v>v_{\theta}^{*}$.

- 1. Consider first the control provided by taking equality in $(\mathbf{A})$, that is

$$
u_{L} \equiv 0, \quad u_{A}(t)=\left|w^{\top} x(t)\right|_{+}
$$

In these conditions, Theorem 6 guarantees that (6) holds for any initial condition $x(0)$, and the values of $u_{A}(t)$ are zero from a certain time and beyond, which certainly implies

$$
\lim _{t \rightarrow+\infty} u_{A}(t)=0 \text {. }
$$


(Notice incidentally that this in turn implies not only (6), but also that $\lim _{t \rightarrow+\infty} x(t)=x_{W}^{*}$.) Let

$$
\bar{u}_{A}:=k_{U}\left(L_{U}^{*}+\left(\gamma_{W}-\gamma_{U}\right) A_{U}^{*}\right) .
$$

Due to the assumption that $k \geqslant \gamma_{W}$, one has $w<_{C} 0$, and it is clear that, for any initial condition $x_{0}$ within $\llbracket x_{U}^{*} ; x_{W}^{*} \rrbracket$, the control law $($ A.8) fulfills

$$
\forall t \geqslant 0, \quad 0 \leqslant u_{A}(t) \leqslant \bar{u}_{A} .
$$

By monotonicity, it is possible to compare the corresponding solution of (2)(A.8) with the trajectory obtained for the system with constant input:

$$
\dot{x}=f(x)+B\left(\begin{array}{c}
0 \\
\bar{u}_{A}
\end{array}\right)
$$

departing from the same initial condition. This makes it possible to affirm that system (A.9) has no equilibrium with positive $L_{U}, A_{U}$.

-2. Similarly, consider now Theorem 5 . One has when $u=L_{U} L_{W}$

$$
\dot{L}_{W}=\gamma_{W} \mathcal{R}_{W} A_{W}-\left(1+L_{W}\right) L_{W}, \quad \dot{A}_{W}=L_{W}-\gamma_{W} A_{W}
$$

and thus $x(t) \leqslant_{C} x_{W}^{*}$ for any $t \geqslant 0$. Let

$$
\bar{u}_{L}:=L_{U}^{*} L_{W}^{*} .
$$

Arguing as before, the control law is such that, for any initial condition in $\llbracket x_{U}^{*}, x_{W}^{*} \rrbracket$

$$
\forall t \geqslant 0, \quad 0 \leqslant u_{L}(t) \leqslant \bar{u}_{L}
$$

and we conclude that the system

$$
\dot{x}=f(x)+B\left(\begin{array}{c}
\bar{u}_{L} \\
0
\end{array}\right)
$$

has no equilibrium with positive $L_{U}, A_{U}$.

- 3. From the two previous points, we deduce that, for any $\theta \in[0,1]$, the system (11) has no equilibrium with positive $L_{U}, A_{U}$ whenever

$$
v \geqslant 2 \max \left\{\frac{\bar{u}_{L}}{\mathcal{R}_{W}} ; \bar{u}_{A}\right\},
$$


because in this case,

$$
\left(\begin{array}{c}
\mathcal{R}_{W} \theta \\
1-\theta
\end{array}\right) v=u \geqslant\left(\begin{array}{c}
0 \\
\bar{u}_{A}
\end{array}\right) \text { if } \theta \in\left[0, \frac{1}{2}\right], \quad u \geqslant\left(\begin{array}{c}
\bar{u}_{L} \\
0
\end{array}\right) \text { if } \theta \in\left[\frac{1}{2}, 1\right] .
$$

The set of those $v>0$ such that (2)-(10) has no equilibrium with positive $L_{U}, A_{U}$ is thus a nonempty up-set ${ }^{5}$ bounded away from 0 , and defining $v_{\theta}^{*}$ as its infimum yields the threshold property indicated in the statement.

- 4. We now characterize the number $v_{\theta}^{*}$. Let $v$ be a nonnegative number and $x$ an equilibrium of the corresponding system, that is:

$$
f(x)+B\left(\begin{array}{c}
\mathcal{R}_{W} \theta \\
1-\theta
\end{array}\right) v=0
$$

or explicitly:

$$
\begin{gathered}
\gamma_{U} \mathcal{R}_{U} \frac{A_{U}}{A_{U}+A_{W}} A_{U}=\left(1+L_{U}+L_{W}\right) L_{U}, \quad \gamma_{U} A_{U}=L_{U} \\
\gamma_{W} \mathcal{R}_{W} A_{W}+\mathcal{R}_{W} \theta v=\left(1+L_{U}+L_{W}\right) L_{W}, \quad \gamma_{W} A_{W}=L_{W}+(1-\theta) v
\end{gathered}
$$

It has been recalled in Theorem 2 that when $v=0,(\mathrm{~A} .10$ has exactly two solutions with positive values of $L_{U}, A_{U}$, corresponding to the Wolbachia-free equilibrium and the coexistence equilibrium.

Suppose now that $v>0$. If $A_{U} \neq 0$, then $L_{U}=\gamma_{U} A_{U} \neq 0$, and

$$
\begin{gathered}
\mathcal{R}_{U} \frac{A_{U}}{A_{U}+A_{W}}=1+L_{U}+L_{W} \\
\mathcal{R}_{W}\left(L_{W}+(1-\theta) v\right)+\mathcal{R}_{W} \theta v=\mathcal{R}_{W}\left(L_{W}+v\right)=\left(1+L_{U}+L_{W}\right) L_{W}
\end{gathered}
$$

Therefore one deduces from (A.11b and the fact that $v>0$ that $L_{W} \neq 0$ and that

$$
\mathcal{R}_{W}\left(1+\frac{v}{L_{W}}\right)=1+L_{U}+L_{W}
$$

\footnotetext{
${ }^{5}$ By definition (see e.g. [51]), a subset $U$ of a partially ordered set $(X, \geqslant)$ is an up-set (or upper set) if, for any $x, y \in X$,

$$
x \in U \text { and } y \geqslant x \Rightarrow y \in U \text {. }
$$
}


from which one expresses $L_{U}$ as a function of $L_{W}$ :

$$
L_{U}=\mathcal{R}_{W}-1+\mathcal{R}_{W} \frac{v}{L_{W}}-L_{W}
$$

On the other hand, eliminating $1+L_{U}+L_{W}$ from A.11a and A.12 yields:

$$
1+\frac{v}{L_{W}}=\frac{\mathcal{R}_{U}}{\mathcal{R}_{W}} \frac{A_{U}}{A_{U}+A_{W}}=\frac{\mathcal{R}_{U}}{\mathcal{R}_{W}} \frac{L_{U}}{L_{U}+\frac{\gamma_{U}}{\gamma_{W}}\left(L_{W}+(1-\theta) v\right)} .
$$

Inserting the value of $L_{U}$ obtained from A.13 in identity $\mathrm{A}$.14 leads to the following equation in $L_{W}$ :

$$
1+\frac{v}{L_{W}}=\frac{\mathcal{R}_{U}}{\mathcal{R}_{W}} \frac{\mathcal{R}_{1}+\mathcal{R}_{W} \frac{v}{L_{W}}-L_{W}}{\mathcal{R}_{1}+\mathcal{R}_{W} \frac{v}{L_{W}}-L_{W}+\frac{\gamma_{U}}{\gamma_{W}}\left(L_{W}+(1-\theta) v\right)},
$$

for $\mathcal{R}_{1}$ defined in $12 \mathrm{~d}$ ).

Introduce for simplicity the new unknown

$$
X:=\frac{v}{L_{W}}
$$

Multiplying numerator and denominator of the right-hand side by $X$, the previous equation becomes:

$$
1+X=\frac{\mathcal{R}_{U}}{\mathcal{R}_{W}} \frac{\mathcal{R}_{1} X+\mathcal{R}_{W} X^{2}-v}{\left(\mathcal{R}_{1}+\frac{\gamma_{U}}{\gamma_{W}}(1-\theta) v\right) X+\mathcal{R}_{W} X^{2}-\left(1-\frac{\gamma_{U}}{\gamma_{W}}\right) v}
$$

or again:

$$
\begin{aligned}
(1+X)\left(\mathcal{R}_{W} X^{2}+\left(\mathcal{R}_{1}+\frac{\gamma_{U}}{\gamma_{W}}(1-\theta) v\right) X\right. & \left.-\left(1-\frac{\gamma_{U}}{\gamma_{W}}\right) v\right) \\
& =\frac{\mathcal{R}_{U}}{\mathcal{R}_{W}}\left(\mathcal{R}_{W} X^{2}+\mathcal{R}_{1} X-v\right)
\end{aligned}
$$

Ordering in powers of $X$, one finally obtains the third order polynomial equation

$$
\mathcal{P}(X):=a X^{3}+b X^{2}+c X+d=0
$$

for the values $a, b, c, d$ defined in $12 \mathrm{~b})-(12 \mathrm{c})$. 
One has thus shown so far that: if, for a given $v>0$, exists an equilibrium with positive $L_{U}, A_{U}$ (that is a solution of (A.11) ), then necessarily $X$ defined in A.15 solves equation A.16). Let us now examine the converse.

- 5. Notice first that, due to (A.14), whose right-hand side varies from 0 to $\frac{\mathcal{R}_{U}}{\mathcal{R}_{W}}$ for $L_{U}>0$, any solution of (A.11) is such that $X=\frac{v}{L_{W}}$ verifies:

$$
1+X<\frac{\mathcal{R}_{U}}{\mathcal{R}_{W}}
$$

that is, with $\mathcal{R}_{2}$ defined in $\left.12 \mathrm{~d}\right)$ :

$$
X<\frac{\mathcal{R}_{2}}{\mathcal{R}_{W}} .
$$

Reciprocally, let $X>0$ be a root of the polynomial $\mathcal{P}$ defined in (A.16), fulfilling (A.17). Define $L_{W}:=\frac{v}{X}>0$. For this value, there exists a unique $L_{U}>0$ such that equality holds between the first and last expressions in (A.14). Then, construct $A_{U}>0, A_{W}>0$ by use of the second parts of A.10a) and (A.10b). The element $\left(L_{U}, A_{U}, L_{W}, A_{W}\right)$ constructed in this way is an equilibrium of (11) with positive $L_{U}, A_{U}$.

Therefore, we have proved so far that: there exists an equilibrium point with positive $L_{U}, A_{U}$ iff there exists a root $X$ of $\mathcal{P}$ defined in (A.16) within the open interval $\left(0, \frac{\mathcal{R}_{2}}{\mathcal{R}_{W}}\right)$.

- 6. For nonzero values of $v$, the coefficient $d=d(v)$ of the polynomial $\mathcal{P}$ is positive, and thus

$$
\forall v>0, \quad \mathcal{P}(0)>0 .
$$


On the other hand,

$$
\begin{aligned}
\mathcal{P}\left(\frac{\mathcal{R}_{2}}{\mathcal{R}_{W}}\right) & a\left(\frac{\mathcal{R}_{2}}{\mathcal{R}_{W}}\right)^{3}+b\left(\frac{\mathcal{R}_{2}}{\mathcal{R}_{W}}\right)^{2}+c\left(\frac{\mathcal{R}_{2}}{\mathcal{R}_{W}}\right)+d \\
= & \mathcal{R}_{W}\left(\frac{\mathcal{R}_{2}}{\mathcal{R}_{W}}\right)^{3}+\left(\mathcal{R}_{1}-\mathcal{R}_{2}+\frac{\gamma_{U}}{\gamma_{W}}(1-\theta) v\right)\left(\frac{\mathcal{R}_{2}}{\mathcal{R}_{W}}\right)^{2} \\
& -\left(\frac{\mathcal{R}_{1} \mathcal{R}_{2}}{\mathcal{R}_{W}}-\left(\frac{\gamma_{U}}{\gamma_{W}}(2-\theta)-1\right) v\right) \frac{\mathcal{R}_{2}}{\mathcal{R}_{W}}+\left(\frac{\gamma_{U}}{\gamma_{W}}+\frac{\mathcal{R}_{2}}{\mathcal{R}_{W}}\right) v \\
= & \left(\frac{1}{\mathcal{R}_{W}}\right)^{2}\left[\mathcal{R}_{2}^{3}+\left(\mathcal{R}_{1}-\mathcal{R}_{2}+\frac{\gamma_{U}}{\gamma_{W}}(1-\theta) v\right) \mathcal{R}_{2}^{2}\right. \\
& \left.-\left(\mathcal{R}_{1} \mathcal{R}_{2}-\left(\frac{\gamma_{U}}{\gamma_{W}}(2-\theta)-1\right) \mathcal{R}_{W} v\right) \mathcal{R}_{2}+\left(\frac{\gamma_{U}}{\gamma_{W}} \mathcal{R}_{W}+\mathcal{R}_{2}\right) \mathcal{R}_{W} v\right] \\
= & \left(\frac{1}{\mathcal{R}_{W}}\right)^{2}\left[\frac{\gamma_{U}}{\gamma_{W}}(1-\theta) \mathcal{R}_{2}^{2}+\left(\frac{\gamma_{U}}{\gamma_{W}}(2-\theta)-1\right) \mathcal{R}_{W} \mathcal{R}_{2}+\left(\frac{\gamma_{U}}{\gamma_{W}} \mathcal{R}_{W}+\mathcal{R}_{2}\right) \mathcal{R}_{W}\right] v .
\end{aligned}
$$

The expression between brackets in the previous line is equal to

$$
\frac{\gamma_{U}}{\gamma_{W}}\left((1-\theta) \mathcal{R}_{2}^{2}+(2-\theta) \mathcal{R}_{W} \mathcal{R}_{2}+\mathcal{R}_{W 2}\right) v>0
$$

Therefore, in addition to A.18a, we also have:

$$
\forall v>0, \quad \mathcal{P}\left(\frac{\mathcal{R}_{2}}{\mathcal{R}_{W}}\right)>0 .
$$

- 7. As mentioned before, the system has two equilibrium points with positive $L_{U}, A_{U}$ for $v=0$. We now show that, for small enough positive $v$, the thirdorder polynomial $\mathcal{P}$ has one root in $(-\infty, 0)$ and two inside the interval $\left(0, \frac{\mathcal{R}_{2}}{\mathcal{R}_{W}}\right)$.

For this, consider a constant $h$ such that

$$
h>\frac{1}{\mathcal{R}_{1}}+\frac{\mathcal{R}_{W}}{\mathcal{R}_{2}} \frac{\gamma_{U}}{\gamma_{W}} .
$$

For small positive $v$, one has

$\mathcal{P}(h v)=a h^{3} v^{3}+b h^{2} v^{2}+c h v+d=c h v+d+o(v)=\left(-\frac{\mathcal{R}_{1} \mathcal{R}_{2}}{\mathcal{R}_{W}} h+\frac{\gamma_{U}}{\gamma_{W}}+\frac{\mathcal{R}_{2}}{\mathcal{R}_{W}}\right) v+o(v)$, 
where by definition $o(v)$ represents a function such that $\frac{o(v)}{v} \rightarrow 0$ when $v \rightarrow$ $0^{+}$.

For small enough positive $v, \mathcal{P}(h v)$ is therefore negative, due to (A.19). For small enough positive $v$, the third-order polynomial $\mathcal{P}(X)$ is thus positive at $X=0$ and at $X=\frac{\mathcal{R}_{2}}{\mathcal{R}_{W}}$, negative at $X=h v$, while in the same time $\lim _{X \rightarrow \pm \infty} \mathcal{P}(X)= \pm \infty$. Whence the announced behavior.

Due to the two inequalities in (A.18), when $v$ increases, the two roots in the interval $\left(0, \frac{\mathcal{R}_{2}}{\mathcal{R}_{W}}\right)$ may only disappear from this interval when colliding, giving rise to a saddle-node bifurcation of the dynamical system. The function $\Delta_{\theta}(v)$ given in (12a) is the discriminant of the cubic equation (A.16), see e.g. [52, Exercice 10.7], and the annihilation of the two roots occurs whenever it vanishes. The quantity $v_{\theta}^{*}$ previously exhibited is thus the smallest positive root of the polynomial $\Delta_{\theta}$.

- 8. We finally show that (6) is verified for any $v>v_{\theta}^{*}>0$. In such a case, any possible equilibrium is such that $L_{U}=A_{U}=0$, and A.10b then simplifies in

$$
\gamma_{W} \mathcal{R}_{W} A_{W}+\mathcal{R}_{W} \theta v=\left(1+L_{W}\right) L_{W}, \quad \gamma_{W} A_{W}=L_{W}+(1-\theta) v .
$$

This implies $\mathcal{R}_{W}\left(L_{W}+v\right)=\left(1+L_{W}\right) L_{W}$, and thus

$$
L_{W}^{2}-\mathcal{R}_{1} L_{W}-\mathcal{R}_{W} v=0 .
$$

A unique, positive, equilibrium $\left(L_{W}^{* *}, A_{W}^{* *}\right)$ is thus obtained for the monotone system

$$
\dot{L}=\gamma_{W} \mathcal{R}_{W} A-(1+L) L+\mathcal{R}_{W} \theta v, \quad \dot{A}=L-\gamma_{W} A+(1-\theta) v,
$$

which is given by:

$$
L_{W}^{* *}=\frac{1}{2}\left(\mathcal{R}_{1}+\sqrt{\mathcal{R}_{1}^{2}+4 \mathcal{R}_{W} v}\right), \quad A_{W}^{* *}=\frac{1}{\gamma_{W}}\left(L_{W}+(1-\theta) v\right) .
$$

Applying [31, Chapter 2, Theorem 3.1, p. 18] as done previously in the proof of Theorem 1, this equilibrium, being unique, is GAS. Moreover, it fulfills evidently:

$$
L_{W}^{* *}>\mathcal{R}_{1}=L_{W}^{*}, \quad A_{W}^{* *}>\frac{1}{\gamma_{W}} L_{W}>\frac{1}{\gamma_{W}} L_{W}^{*}=A_{W}^{*} .
$$

Applying comparison demonstrates finally that (6) is fulfilled on any trajectory. This achieves the demonstration of Proposition 8 . 
Appendix A.7. Proof of Theorem 9

- 1. When $(\mathbf{L}$ ' $)$ holds, then

$$
\begin{gathered}
\dot{L}_{U}=\gamma_{U} \mathcal{R}_{U} \frac{A_{U}}{A_{U}+A_{W}} A_{U}-\left(1+L_{U}+L_{W}\right) L_{U} \\
\dot{A}_{U}=L_{U}-\gamma_{U} A_{U} \\
\dot{L}_{W}>\min \left\{\gamma_{W} \mathcal{R}_{W} A_{W}-\left(1+L_{U}+L_{W}\right) L_{W}+v_{1}^{*}, \gamma_{W} \mathcal{R}_{W} A_{W}-\left(1+L_{W}\right) L_{W}\right\}, \\
\dot{A}_{W} \geqslant L_{W}-\gamma_{W} A_{W}
\end{gathered}
$$

Let $f_{L}$ be the function defined by the right-hand side of $\mathrm{A} .20$, that is to say

$$
f_{L}(x):=f(x)+\left(\begin{array}{llll}
0 & 0 & 1 & 0
\end{array}\right)^{\top} \min \left\{u_{1}^{* *}, L_{U} L_{W}\right\} .
$$

This function is locally Lipschitz continuous and continuously differentiable almost everywhere. Notice that it shares the same monotonicity and irreducibility properties than the two functions of which it is the minimum. In particular, the system

$$
\dot{x^{\prime}}=f_{L}\left(x^{\prime}\right)
$$

is SOP for the ordering defined in (5).

For any initial condition $x(0)$ in the set $\mathcal{S}$ defined in Theorem 4 , one has

$$
\dot{x} \geqslant f_{L}(x), \quad x(0) \geqslant_{C} x_{U}^{*},
$$

and due to the monotonicity property of (A.21), one deduces

$$
\forall t \geqslant T, \quad x(t) \geqslant_{C} x^{\prime}(t)
$$

where $x^{\prime}$ is the solution of A.21 initialized at $x^{\prime}(0)=x_{U}^{*}$. In order to obtain (6) for any initial condition, it is thus sufficient to prove this property for this specific trajectory.

- 2. Let us thus study the asymptotic behaviour of the solution $x^{\prime}$ of (A.21) departing from $x^{\prime}(0)=x_{U}^{*}$. As enunciated in Proposition 8, the system

$$
\dot{x}=f(x)+\left(\begin{array}{llll}
0 & 0 & 1 & 0
\end{array}\right)^{\top} u_{1}^{* *}
$$

has a unique equilibrium, for which $L_{U}, A_{U}$ are null. On the other hand, it is easy to establish that the system

$$
\dot{x}=f(x)+\left(\begin{array}{llll}
0 & 0 & 1 & 0
\end{array}\right)^{\top} L_{U} L_{W}
$$


has exactly three equilibrium points, namely $x_{W}^{*}, x_{U}^{*}$ and $(0,0,0,0)$. The first one is locally asymptotically stable, while the two other ones are unstable. Clearly, every equilibrium point for A.21 has to be equilibrium for one of the two systems A.22 or A.23.

At the equilibrium point $x_{U}^{*}$, one has by definition $f_{L}\left(x_{U}^{*}\right)=0$. On the other hand, only nonnegative control values are possible, therefore $f_{L}\left(x^{\prime}(T)\right) \geqslant_{C}$ 0 for any $t \geqslant 0$, see [31, Chapter 3, Proposition 2.1, p. 34]. Due to the position of $x^{\prime}(0)=x_{U}^{*}$ on the boundary $\partial \mathcal{S}$ of $\mathcal{S}$, the first two components of $x^{\prime}$ cannot increase, while its last two components cannot decrease. Therefore, when $u_{L}$ ceases to be zero, there exists a time $T \geqslant 0$ at which

$$
f_{L}\left(x^{\prime}(T)\right)>_{C} 0
$$

Due to [31, Chapter 3, Proposition 2.1, p. 34], one deduces that $f_{L}\left(x^{\prime}(t)\right)>_{C}$ 0 for any $t \geqslant T$ and the trajectory thus converges towards an equilibrium point distinct from $x_{U}^{*}$. As the system is SOP, $x^{\prime}(t)$ lies indeed in the interior of $\mathcal{S}$ for $t>T$, and therefore this equilibrium cannot be $(0,0,0,0)$. There thus remains two possible values for limit, namely the nonzero fully infected equilibrium points of $\mathrm{A} .22$ and $\mathrm{A} .23$. To decipher which of them is the actual limit, notice that $w^{\top} x$ is negative at these two points, so the value of the switching signal is null in both of them. Therefore system $(\mathrm{A} .23$ is the active mode of A.20 in these points, and the equilibrium of (A.22) is not an equilibrium of (A.20). This shows finally that the solution $x^{\prime}$ of $(\mathrm{A} .21)$ departing from $x^{\prime}(0)=x_{U}^{*}$ converges towards $x_{W}^{*}$.

By the comparison argument already mentioned, one obtains that (8) is fulfilled for the trajectory of A.20 initialized in $x_{U}^{*}$, that is (6) (see Remark 1). As a conclusion, all trajectories converge towards $x_{W}^{*}$ asymptotically.

The last property of the statement, i.e. that (6) holds with equality if $u_{L}, u_{A}$ vanish when $t \rightarrow+\infty$, is straightforward.

\section{References}

[1] L. Alphey, M. Benedict, R. Bellini, G. G. Clark, D. A. Dame, M. W. Service, S. L. Dobson, Sterile-Insect Methods for Control of MosquitoBorne Diseases: An Analysis, Vector-Borne and Zoonotic Diseases 10 (3) (2010) 295-311.

[2] L. Alphey, Genetic control of mosquitoes, Annual review of entomology 59. 
[3] C. J. McMeniman, R. V. Lane, B. N. Cass, A. W. Fong, M. Sidhu, Y.-F. Wang, S. L. O'neill, Stable introduction of a life-shortening Wolbachia infection into the mosquito Aedes aegypti, Science 323 (5910) (2009) 141-144.

[4] L. A. Moreira, I. Iturbe-Ormaetxe, J. A. Jeffery, G. Lu, A. T. Pyke, L. M. Hedges, B. C. Rocha, S. Hall-Mendelin, A. Day, M. Riegler, L. E. Hugo, K. N. Johnson, B. H. Kay, E. A. McGraw, A. F. van den Hurk, P. A. Ryan, S. L. O'Neill, A Wolbachia Symbiont in Aedes aegypti Limits Infection with Dengue, Chikungunya, and Plasmodium, Cell 139 (7) (2009) 1268-1278.

[5] A. A. Hoffmann, B. L. Montgomery, J. Popovici, I. Iturbe-Ormaetxe, P. H. Johnson, F. Muzzi, M. Greenfield, M. Durkan, Y. S. Leong, Y. Dong, H. Cook, J. Axford, A. G. Callahan, N. Kenny, C. Omodei, E. A. McGraw, P. A. Ryan, S. A. Ritchie, M. Turelli, S. L. O'Neill, Successful establishment of Wolbachia in Aedes populations to suppress dengue transmission, Nature 476 (7361) (2011) 454-457.

[6] A. Wilder-Smith, D. J. Gubler, S. C. Weaver, T. P. Monath, D. L. Heymann, T. W. Scott, Epidemic arboviral diseases: priorities for research and public health, The Lancet infectious diseases 17 (3) (2017) e101-e106.

[7] M. J. Keeling, F. Jiggins, J. M. Read, The invasion and coexistence of competing Wolbachia strains, Heredity 91 (4) (2003) 382.

[8] J. Farkas, P. Hinow, Structured and unstructured continuous models for Wolbachia infections, Bulletin of mathematical biology 72 (8) (2010) 2067-2088.

[9] B. Zheng, M. Tang, J. Yu, Modeling Wolbachia spread in mosquitoes through delay differential equations, SIAM Journal on Applied Mathematics 74 (3) (2014) 743-770.

[10] M. A. H. B. da Silva, G. Sallet, Monotone dynamical systems and some models of Wolbachia in Aedes Aegypti populations, Revue Africaine de la Recherche en Informatique et Mathématiques Appliquées 20. 
[11] B. Zheng, Y. Xiao, A mathematical model to assess the effect of the constant release policy on population suppression, Nonlinear Analysis and Differential Equations 5 (4) (2017) 197-207.

[12] L. Yakob, S. Funk, A. Camacho, O. Brady, W. J. Edmunds, Aedes aegypti control through modernized, integrated vector management, PLoS currents 9.

[13] L. Xue, C. A. Manore, P. Thongsripong, J. M. Hyman, Two-sex mosquito model for the persistence of Wolbachia, Journal of biological dynamics 11 (sup1) (2017) 216-237.

[14] D. E. Campo-Duarte, D. Cardona-Salgado, O. Vasilieva, Establishing wMelPop Wolbachia infection among wild Aedes aegypti females by optimal control approach, Appl Math Inf Sci 11 (4) (2017) 1011-1027.

[15] D. E. Campo-Duarte, O. Vasilieva, D. Cardona-Salgado, M. Svinin, Optimal control approach for establishing wMelPop Wolbachia infection among wild Aedes aegypti populations, Journal of mathematical biology 76 (7) (2018) 1907-1950.

[16] P.-A. Bliman, M. S. Aronna, F. C. Coelho, M. A. Da Silva, Ensuring successful introduction of Wolbachia in natural populations of Aedes aegypti by means of feedback control, Journal of mathematical biology 76 (5) (2018) 1269-1300.

[17] L. Almeida, Y. Privat, M. Strugarek, N. Vauchelet, Optimal releases for population replacement strategies, application to Wolbachia, working paper or preprint (Jun. 2018).

URL https://hal . archives-ouvertes.fr/hal-01807624

[18] H. Hughes, N. F. Britton, Modelling the use of Wolbachia to control dengue fever transmission, Bulletin of mathematical biology 75 (5) (2013) 796-818.

[19] J. Koiller, M. Da Silva, M. Souza, C. Codeço, A. Iggidr, G. Sallet, Aedes, Wolbachia and Dengue, Research Report RR-8462, Inria, France (Jan. 2014). 
[20] M. Z. Ndii, R. I. Hickson, D. Allingham, G. Mercer, Modelling the transmission dynamics of dengue in the presence of Wolbachia, Mathematical biosciences 262 (2015) 157-166.

[21] D. A. Focks, et al., A review of entomological sampling methods and indicators for dengue vectors, Tech. rep., Geneva: World Health Organization (2004).

[22] J. B. Silver, Mosquito ecology: field sampling methods, Springer Science \& Business Media, 2007.

[23] L. Lacey, R. Frutos, H. Kaya, P. Vail, Insect pathogens as biological control agents: Do they have a future?, Biological Control 21. doi:10.1006/bcon.2001.0938.

URL http://gen.lib.rus.ec/scimag/index.php?s=10.1006/bcon. 2001.0938

[24] C. L. Brelsfoard, S. L. Dobson, Wolbachia-based strategies to control insect pests and disease vectors, Asia Pac. J. Mol. Biol. Biotechnol 17 (3) (2009) 55-63.

[25] G. Benelli, C. L. Jeffries, T. Walker, Biological control of mosquito vectors: past, present, and future, Insects 7 (4) (2016) 52.

[26] N. L. Achee, J. P. Grieco, H. Vatandoost, G. Seixas, J. Pinto, L. ChingNg, A. J. Martins, W. Juntarajumnong, V. Corbel, C. Gouagna, et al., Alternative strategies for mosquito-borne arbovirus control, PLoS neglected tropical diseases $13(1)$.

[27] M. Moisan, O. Bernard, J.-L. Gouzé, Near optimal interval observers bundle for uncertain bioreactors, Automatica 45 (1) (2009) 291-295.

[28] D. Efimov, T. Raïssi, S. Chebotarev, A. Zolghadri, Interval state observer for nonlinear time varying systems, Automatica 49 (1) (2013) 200-205.

[29] D. Efimov, T. Raïssi, Design of interval observers for uncertain dynamical systems, Automation and Remote Control 77 (2) (2016) 191-225.

[30] M. W. Hirsch, Stability and convergence in strongly monotone dynamical systems, J. reine angew. Math 383 (1988) 1-53. 
[31] H. L. Smith, Monotone dynamical systems: an introduction to the theory of competitive and cooperative systems, Vol. 41 of Mathematical surveys and monographs, American Mathematical Society, 1995.

[32] P.-A. Bliman, Feedback control principles for biological control of dengue vectors, in: Proceedings of European Control Conference, Naples (Italy), 2019 .

[33] H. L. Yeap, P. Mee, T. Walker, A. R. Weeks, S. L. O'Neill, P. Johnson, S. A. Ritchie, K. M. Richardson, C. Doig, N. M. Endersby, et al., Dynamics of the "popcorn" Wolbachia infection in outbred Aedes aegypti informs prospects for mosquito vector control, Genetics 187 (2) (2011) $583-595$.

[34] T. Ruang-Areerate, P. Kittayapong, Wolbachia transinfection in Aedes aegypti: a potential gene driver of dengue vectors, Proceedings of the National Academy of Sciences 103 (33) (2006) 12534-12539.

[35] T. Southwood, G. Murdie, M. Yasuno, R. J. Tonn, P. Reader, Studies on the life budget of Aedes aegypti in Wat Samphaya, Bangkok, Thailand, Bulletin of the World Health Organization 46 (2) (1972) 211.

[36] D. A. Focks, D. Haile, E. Daniels, G. A. Mount, Dynamic life table model for Aedes aegypti (Diptera: Culicidae): analysis of the literature and model development, Journal of medical entomology 30 (6) (1993) 1003-1017.

[37] S. L. O'Neill, A. A. Hoffman, J. H. Werren (Eds.), Influential Passengers: Inherited Microorganisms and Arthropod Reproduction, Oxford University Press, 1998.

[38] T. Walker, P. Johnson, L. Moreira, I. Iturbe-Ormaetxe, F. Frentiu, C. McMeniman, Y. S. Leong, Y. Dong, J. Axford, P. Kriesner, et al., The $w$ Mel Wolbachia strain blocks dengue and invades caged Aedes aegypti populations, Nature 476 (7361) (2011) 450-453.

[39] H. L. Smith, Monotone dynamical systems: Reflections on new advances \& applications, Discrete \& Continuous Dynamical Systems-A 37 (1) (2017) 485-504. 
[40] D. Angeli, E. D. Sontag, Monotone control systems, IEEE Transactions on Automatic Control 48 (2003) 1684-1698.

[41] P.-A. Bliman, Feedback control principles for biological control of dengue vectors, arXiv preprint arXiv:1903.00730.

[42] U. Itkis, Control systems of variable structure, Wiley New York, 1976.

[43] V. I. Utkin, Sliding Modes in Control and Optimization, SpringerVerlag, 1992.

[44] W. A. Coppel, Stability and asymptotic behavior of differential equations, Heath mathematical monographs, Heath, 1965.

[45] P.-A. Bliman, D. Cardona-Salgado, Y. Dumont, O. Vasilieva, Implementation of control strategies for sterile insect techniques, Mathematical biosciences 314 (2019) 43-60.

[46] J.-L. Gouzé, A criterion of global convergence to equilibrium for differential systems with an application to Lotka-Volterra systems, Research report 0894, Inria, France (1988).

[47] D. Angeli, P. D. Leenheer, E. D. Sontag, A small-gain theorem for almost global convergence of monotone systems, Systems \& Control Letters 52 (5) (2004) 407-414.

[48] M. Malisoff, P. D. Leenheer, A small-gain theorem for monotone systems with multi-valued input-state characteristics, IEEE Transactions on Automatic Control 41 (2) (2006) 287-292.

[49] T. Gedeon, G. Hines, Multi-valued characteristics and Morse decompositions, Journal of Differential Equations 247 (4) (2009) 1013-1042.

[50] G. A. Enciso, Fixed points and convergence in monotone systems under positive or negative feedback, International Journal of Control 87 (2) (2014) 301-311.

[51] B. A. Davey, H. A. Priestley, Introduction to lattices and order, Cambridge university press, 2002.

[52] R. S. Irving, Integers, polynomials, and rings: a course in algebra, Springer Science \& Business Media, 2003. 